\title{
Linking Numbers, Contacts, and Mutual Inductances of a Random Set of Closed Curves
}

\section{B. Duplantier}

Service de Physique Théorique, CEN-Saclay, Boite Postale No. 2, F-91190 Gif-sur-Yvette, France

\begin{abstract}
We establish here a new, general result of integral geometry, concerning closed rigid curves of arbitrary shapes in $E^{3}$ and their linking numbers $I$. It generalizes by a different method, the interesting integral property of $I^{2}$ found recently by Pohl and extended by des Cloizeaux and Ball, for two curves. We consider $n$ closed curves linked successively to each other and forming a ring. The cyclic product of their linking numbers is integrated over the group of rigid motions of the curves. This integral is shown to factorize over a special algebra of characteristic functions. Each curve possesses two such intrinsic functions. The same algebra is shown to describe a larger class of integral geometry properties: a new theorem is established for a family of displacement integrals involving linking numbers, contact angles, and mutual inductances of the set of $n$ curves.
\end{abstract}

\section{Introduction}

Topology of knotting and linking of closed curves in three-dimensional space involves very interesting mathematical problems. Two configurations of a set of closed curves are said to be topologically equivalent if one can transform continuously one into the other without forming any double point, (or opening one curve). The main problem is therefore to differentiate the irreducible configuration classes. This subject is also important in polymer theory. Polymer chains forming rings can indeed be considered ideally as closed solid curves. They are free to change their shape but not to open. Thus a given set of polymer rings may not develop new linkings by a simple continuous change of configuration. The statistical mechanics of a set of rings is then defined in a phase space restricted by topological constraints. Any progress in mathematical description of the linking of closed curves could be very useful.

Several topological invariants have been proposed for distinguishing whether or not two curves are linked together. In general these invariants do not yield a complete topological description. Two configurations with different values of 


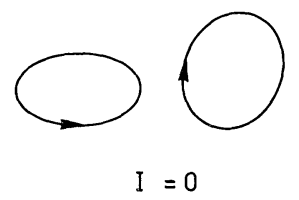

(a)

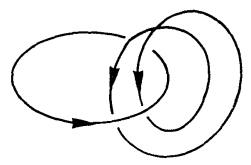

$I=-2$

(c)

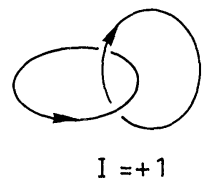

(b)

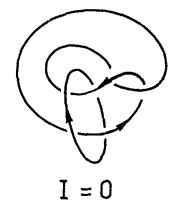

(d)

Fig. 1a-d. Algebraic linking numbers $I$ of closed curves

invariant are necessarily irreducible one to another, but the converse is not necessarily true. In this article we shall consider a very simple invariant due to Gauss [1].

The Gauss linking number $I_{12}$ of two closed curves $\gamma_{1}, \gamma_{2}$ is defined by the double curvilinear integral ${ }^{1}[1]$

$$
I_{12}=-\oint_{\gamma_{1}} \oint_{\gamma_{2}}\left(\nabla_{1} \frac{1}{\left|\mathbf{r}_{1}-\mathbf{r}_{2}\right|}, d \mathbf{r}_{1}, d \mathbf{r}_{2}\right),
$$

where $\mathbf{r}_{1}$ and $\mathbf{r}_{2}$ denote the positions of two generic points on $\gamma_{1}$ and $\gamma_{2}$ respectively.

This invariant is integer-valued and measures loosely the number of times one such curve winds around the other. More precisely it counts the algebraic number of times one such curve, closed and oriented, crosses the oriented surface, the boundary of which is formed by the other oriented curve (Fig. 1). The sign of integral (1.1) depends on the orientations of these curves. Moreover the quantity $I_{12}$ is symmetric under exchange of 1 and 2

$$
I_{12}=I_{21} \text {. }
$$

Of course, there are topologically linked configurations with $I=0$ (Fig. 1d).

It is worth noting that the Gauss invariant is in fact intimately related to potential theory [2]: the Newtonian potential $\frac{1}{\left|\mathbf{r}_{1}-\mathbf{r}_{2}\right|}[$ which satisfies $\left.\Delta \frac{1}{\left|\mathbf{r}_{1}-\mathbf{r}_{2}\right|}=-4 \pi \delta\left(\mathbf{r}_{1}-\mathbf{r}_{2}\right)\right]$ appears in (1.1). The importance of potential theory in this study of integral geometry will appear clearly later on.

Concerning Gauss' linking number $I$, a quite interesting integral property of the square of $I$ has been very recently found by Pohl [3]. He considered the

1 Throughout this article, we use the notation $(\mathbf{a}, \mathbf{b}, \mathbf{c})=(\mathbf{a} \wedge \mathbf{b}) \cdot \mathbf{c}$ 
integral of $I^{2}$ on the group of relative translations and rotations of the two rigid curves $\gamma_{1}$ and $\gamma_{2}$ (i.e. the "kinematic" integral over the group of Euclidean motions)

$$
\mathscr{I}=\int d^{3} \varrho \frac{d \mathcal{O}}{8 \pi^{2}} I_{12}^{2},
$$

$\varrho$ and $\mathcal{O}$ denote respectively the relative position and orientation of $\gamma_{1}$ and $\gamma_{2}$. He has shown that $\mathscr{I}$ can be written

$$
\mathscr{I}=2 \pi \int_{0}^{\infty} d r \mathscr{A}_{1}(r) \mathscr{A}_{2}(r)
$$

$r$ is a variable with the dimension of a length, and $\mathscr{A}_{1}, \mathscr{A}_{2}$ are two characteristic functions associated with curves $\gamma_{1}$ and $\gamma_{2}$ respectively.

The result (1.4) was proved by Pohl for two plane and convex curves, Des Cloizeaux and Ball [4] recently obtained Pohl's formula (1.4), plus a second term. The latter involves a second characteristic function $\mathscr{B}$, which exists for twisted curves.

The aim of this article is to give a new general theorem on the random linking of a set of $n$ curves, of which Pohl's result is a particular case. We consider $n$ closed curves, arranged as successive links of a ring. We take the product of the successive linking numbers of these $n$ curves, and calculate its integral over the group of rigid motions of the curves in $E^{3}$. We obtain the "factorization" of the integral in terms of characteristic functions $\mathscr{A}, \mathscr{B}$ by using special algebraic rules. Our method is essentially tensorial and uses potential theory. Associated functions $\mathscr{A}, \mathscr{B}$ are here easily obtained from Gauss' theorem.

Further generalizations of Pohl's result are given. Beside the linking numbers, we consider the contacts of the closed curves. They are defined by a certain localized distribution which forces the curves to touch each other. We introduce also the Neumann potentials of the curves [5]. They represent the magnetic interaction energy due to the mutual inductions of the curves [5]. We show that any cyclic product of Gauss invariants, contacts, and mutual inductances can be integrated over random rigid motions of the curves. A general theorem gives this family of kinematic integrals for a set of $n$ arbitrary curves. These integrals can be factorized entirely in terms of the same characteristic functions $\mathscr{A}$ and $\mathscr{B}$, by using special algebraic rules. The real reason for the existence of such results can be understood from the formalism we use here. It will appear in particular that these studies are related to topology, and more essentially to potential theory.

In Sect. 2, a general tensorial description of the linking of $n$ mobile curves is introduced. The characteristic functions $\mathscr{A}, \mathscr{B}$ are defined in Sect. 3. In Sect. 4, Pohl's formula of $[3,4]$ for two curves is obtained from our method. Section 5 gives the general factorization theorem for $n$ closed curves. Section 6 extends it to mutual inductions and contacts. In Sect. 7, the set of explicit formulae for mutual induction, contacts, and linking of two curves is given.

\section{Linking of $\boldsymbol{n}$ Mobile Closed Curves}

Consider $n$ arbitrary closed curves $\gamma_{j}$, labelled by an index $j, j=1, \ldots, n$. Each curve is oriented. On each curve $\gamma_{j}$ are defined two generic points $A_{j}, A_{j}^{\prime}$. Their position 


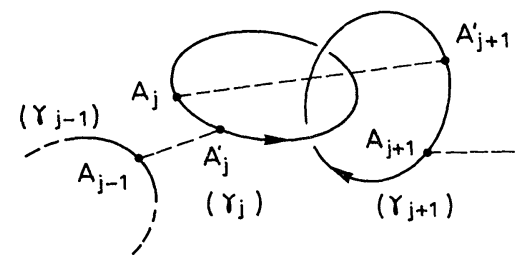

Fig. 2. Generic points along curves $\gamma_{j}$ and $\gamma_{j+1}$ and their association

vectors in three-dimensional space are denoted by $\mathbf{r}_{A_{j}}, \mathbf{r}_{A_{j+1}^{\prime}}$. The Gauss algebraic linking number of the curves $\gamma_{j}$ and $\gamma_{j+1}$ is defined by the integral:

$$
I_{j j+1}=-\frac{1}{4 \pi} \oint_{\gamma_{j}} \oint_{\gamma_{j+1}}\left(\nabla_{A_{j}}\left(\frac{1}{r_{A_{j} A_{j+1}^{\prime}}}\right), d \mathbf{r}_{A_{j}}, d \mathbf{r}_{A_{j+1}^{\prime}}\right) .
$$

We have choosen for convenience to associate together the generic points $A_{j}$ and $A_{j+1}^{\prime}$ for the calculation of the invariant $I_{j j+1}$ (Fig. 2). $r_{A_{j} A_{j+1}^{\prime}}$ is the modulus of the vector defined, by convention, as

$$
\mathbf{r}_{A_{j} A_{j+1}^{\prime}}=\mathbf{r}_{A_{j}}-\mathbf{r}_{A_{j+1}^{\prime}+1} .
$$

The gradient is taken with respect to $\mathbf{r}_{\boldsymbol{A}_{j}}$. The linking number (2.1) depends implicitly on the relative position of the curves $\gamma_{j}$ and $\gamma_{j+1}$, and on their orientations in space. The relative displacement of the pair of curves $\left(\gamma_{j}, \gamma_{j+1}\right)$ is measured by a vector $\boldsymbol{\varrho}_{j}$, joining the origins of $\gamma_{j}$ and $\gamma_{j+1}$. There are $n$ such vectors $\mathbf{Q}_{j}, j=1, \ldots, n$, related by:

$$
\mathbf{\varrho}_{1}+\ldots+\mathbf{\varrho}_{n}=\mathbf{0}
$$

Each curve $\gamma_{j}$ is considered here as a rigid body, that is as a solid in threedimensional space. The set of Euler angles, which defines its orientation, is noted $\mathcal{O}_{j}$. Its total measure is equal to

$$
\int d \mathcal{O}_{j}=8 \pi^{2}
$$

According to these definitions, the linking number (2.1) is in fact a function of $\mathbf{\varrho}_{j}$ and $\mathcal{O}_{j}, \mathcal{O}_{j+1}$ :

$$
I_{j j+1}=I_{j j+1}\left(\boldsymbol{\varrho}_{j}, \mathcal{O}_{j}, \mathcal{O}_{j+1}\right) .
$$

Let us introduce the cyclic product of the successive linking numbers :

$$
\prod_{j=1}^{n} I_{j j+1}=I_{12} I_{23} \ldots I_{n 1}
$$

where, by convention:

$$
n+1 \equiv 1
$$

The only configurations of the curves which give a non-vanishing value for the product (2.6), are necessarily closed chains. These chains are made of the curves $\gamma_{j}$, 


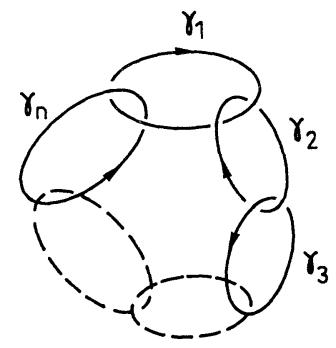

(a)

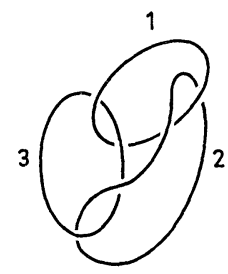

$I_{12} I_{23} I_{31} \neq 0$

(b)

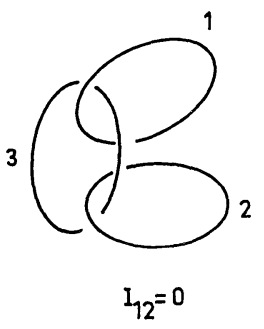

(c)

Fig. 3. a Ring configuration of $n$ closed curves. b A configuration giving a non-zero contribution. $\mathbf{c}$ An open configuration giving a vanishing contribution

linked up together in the order $j=1$ to $n$ (Fig. 3) $)^{2}$. For two curves, the product (2.6) can be written

$$
I_{12} I_{21}=\left(I_{12}\right)^{2}
$$

according to the symmetry property (1.2). For the ordered set of curves $\gamma_{j}$, we consider the kinematic integral $\mathscr{I}$ over all independent relative positions $\boldsymbol{\varrho}_{j}$ $(j=1, \ldots, n-1)$ and upon the set of orientations $\mathcal{O}_{j}(j=1, \ldots, n)$ :

$\mathscr{I}\left(\gamma_{1}, \ldots, \gamma_{n}\right)=\frac{1}{\left(8 \pi^{2}\right)^{n}} \int d^{3} \varrho_{1} \ldots d^{3} \varrho_{n} d \mathcal{O}_{1} \ldots d \mathcal{O}_{n} \delta\left(\varrho_{1}+\ldots+\mathbf{\varrho}_{n}\right) \prod_{j=1}^{n} I_{j j+1}\left(\boldsymbol{\varrho}_{j}, \mathcal{O}_{j}, \mathcal{O}_{j+1}\right)$.

For two curves $\gamma_{1}, \gamma_{2}$ this integral reduces itself to the single integral [see (2.8)]:

$$
\mathscr{I}\left(\gamma_{1}, \gamma_{2}\right)=\frac{1}{\left(8 \pi^{2}\right)^{2}} \int d^{3} \varrho d \mathcal{O}_{1} d \mathcal{O}_{2} I_{12}^{2}\left(\varrho, \mathcal{O}_{1}, \mathcal{O}_{2}\right),
$$

which is the quantity considered by Pohl [3]. Let us now transform the integral (2.9). It is convenient to introduce the differential tensor

$$
d \underset{\sim}{\Gamma}(\mathbf{r})=-\frac{1}{4 \pi} \frac{d \mathbf{r}_{A_{j}} \otimes d \mathbf{r}_{A_{j}^{\prime}}}{|\mathbf{r}|} .
$$

2 Of course, two non-successive curves $j$ and $i$ with $|i-j| \neq 1$, can also be linked, but this linking does not appear in the product (2.6) 
The symbol $\otimes$ represents the tensorial product of three-dimensional vectors. In particular

$$
d \underset{\sim}{\Gamma}\left(\mathbf{r}_{A_{j}}-\mathbf{r}_{A_{j}^{\prime}+1}\right)=-\frac{1}{4 \pi} \frac{d \mathbf{r}_{A_{j}} \otimes d \mathbf{r}_{A_{j}^{\prime}}}{\left|\mathbf{r}_{A_{j}}-\mathbf{r}_{A_{j+1}^{\prime}}\right|} .
$$

Both infinitesimal vectors are tangent to the curve $\gamma_{j}$. The denominator represents the distance between two points belonging respectively to $\gamma_{j}$ and $\gamma_{j+1}$. Let us consider the curl of the differential tensor $d \Gamma$. This operator curl acts on left space indices and on vector $\mathbf{r}_{A_{j}}$ :

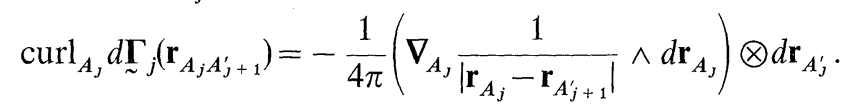

It is convenient at this point to use the following formula of vectorial calculus:

$$
\begin{aligned}
\prod_{j=1}^{n}\left(\nabla_{j}, \mathbf{a}_{j}, \mathbf{a}_{j+1}^{\prime}\right) & =\operatorname{tr}\left\{\left[\left(\nabla_{n} \wedge \mathbf{a}_{n}\right) \otimes \mathbf{a}_{n}^{\prime}\right] \ldots\left[\left(\nabla_{2} \wedge \mathbf{a}_{2}\right) \otimes \mathbf{a}_{2}^{\prime}\right]\left[\left(\nabla_{1} \wedge \mathbf{a}_{1}\right) \otimes \mathbf{a}_{1}^{\prime}\right]\right\} \\
& =\operatorname{tr}\left\{\prod_{j=n}^{1}\left[\left(\nabla_{j} \wedge \mathbf{a}_{j}\right) \otimes \mathbf{a}_{j}^{\prime}\right]\right\}
\end{aligned}
$$

where the $\nabla_{j}, \mathbf{a}_{j}, \mathbf{a}_{j}^{\prime \prime s}(j=1, \ldots, n)$ represent arbitrary vectors in $E^{3}$. One must note the inversion of the order of the indices $j$ in the product of tensors (2.14). The linking numbers (2.1) have the same structure as the factors of the left hand side in (2.14), while a tensorial factor in the right hand side in (2.14) corresponds exactly to the curl (2.13). We can therefore directly write:

$$
\prod_{j=1}^{n} I_{j j+1}=\oint_{\gamma_{n}} \oint_{\gamma_{n}} \ldots \oint_{\gamma_{1}} \oint_{\gamma_{1}} \operatorname{tr}\left\{\prod_{j=n}^{1}\left[\operatorname{curl}_{A_{j}} d \underset{\sim}{\boldsymbol{\Gamma}_{j}}\left(\mathbf{r}_{A_{j} A_{j+1}^{\prime}}\right)\right]\right\} \text {. }
$$

In the right hand side of $(2.15)$, the trace acts on a product of tensors. It is to be noted that in this last formula the successive curves $\gamma_{j}, \gamma_{j+1} \ldots$ have been disentangled with respect to the differential vectors $d \mathbf{r}_{A_{j}}, d \mathbf{r}_{A_{j}^{\prime}}[\operatorname{see}(2.11)]$. It remains to disentangle them with respect to the internal relative vectors $\mathbf{r}_{A_{j}}-\mathbf{r}_{A_{j+1}^{\prime}}$.

This can be done by performing the integration over the relative translation vectors $\mathbf{\varrho}_{j}$ joining curves $\gamma_{j}$ and $\gamma_{j+1}$. Under such a relative translation, $\mathbf{r}_{A_{j} A_{j+1}^{\prime}}$ is transformed:

$$
\mathbf{r}_{A_{j} A_{j+1}^{\prime}}=\mathbf{r}_{A_{j}}-\mathbf{r}_{A_{j+1}^{\prime}} \rightarrow \mathbf{r}_{A_{J}}-\mathbf{r}_{A_{j+1}^{\prime}}+\mathbf{Q}_{j}
$$

Therefore the product (2.15) containing these transformed vectors is

$$
\prod_{j=1}^{n} I_{j j+1}\left(\mathbf{\varrho}_{j}, \mathcal{O}_{j}, \mathcal{O}_{j+1}\right)=\oint_{\gamma_{n}} \oint_{\gamma_{n}} \ldots \oint_{\gamma_{1}} \oint_{\gamma_{1}} \operatorname{tr}\left\{\prod_{j=n}^{1}\left[\operatorname{curl}_{A_{j}} d \underset{\sim}{\boldsymbol{\Gamma}}\left(\mathbf{r}_{A_{j} A_{j+1}^{\prime}}+\mathbf{\varrho}_{j}\right)\right]\right\} .
$$

We then perform a change of variable $\mathbf{Q}_{j} \rightarrow \mathbf{r}_{j}$, by setting, for $j=1, \ldots, n$ :

$$
\mathbf{Q}_{j}=\mathbf{r}_{j}+\mathbf{r}_{A_{j}^{\prime}+1}-\mathbf{r}_{A_{j}^{\prime}} .
$$

For $j=n ; j+1=n+1 \equiv 1$. The $\mathbf{r}_{j}$ 's are $n$ new vectors. This change of variable is local because it involves the $\mathbf{r}_{A_{j}^{\prime}}$ 's, and it is meant to operate inside the curvilinear 
integrals. The Jacobian of the transformation is equal to 1. By summing up each side of Eq. (2.18), we immediately get

$$
\sum_{j=1}^{n} \mathbf{Q}_{j}=\sum_{j=1}^{n} \mathbf{r}_{j}
$$

and thus:

$$
d^{3} \varrho_{1} \ldots d^{3} \varrho_{n} \delta\left(\mathbf{Q}_{1}+\ldots+\underline{\mathbf{Q}}_{n}\right)=d^{3} r_{1} \ldots d^{3} r_{n} \delta\left(\mathbf{r}_{1}+\ldots+\mathbf{r}_{n}\right) .
$$

The effect of this local transformation is to disentangle the successive curves $\gamma_{j}, \gamma_{j+1}$ for all $j=1, \ldots, n$. Indeed, Eqs. (2.16) and (2.18) result in

$$
\mathbf{r}_{A_{j}}-\mathbf{r}_{A_{j+1}^{\prime}+1}+\mathbf{Q}_{j}=\mathbf{r}_{A_{j}}-\mathbf{r}_{A_{j}^{\prime}}+\mathbf{r}_{j}=\mathbf{r}_{j}-\mathbf{r}_{A_{j}^{\prime} A_{j}},
$$

where by convention:

$$
\mathbf{r}_{A_{j}^{\prime} A_{j}}=\mathbf{r}_{A_{j}^{\prime}}-\mathbf{r}_{A_{j}} \text {. }
$$

We note that the last expression in (2.21) is a linear combination of vectors, belonging to curve $\gamma_{j}$ only, with an external variable vector $\mathbf{r}_{j}$.

The tensor $d \underset{\sim}{\boldsymbol{\Gamma}}$ appearing in (2.17) is therefore equal to

$$
d \underset{\sim}{\mathbf{\Gamma}}\left(\mathbf{r}_{A_{j} A_{j+1}^{\prime}+1}+\mathbf{Q}_{j}\right)=d \underset{\sim}{\boldsymbol{\Gamma}}\left(\mathbf{r}_{j}-\mathbf{r}_{A_{j}^{\prime} A_{j}}\right) .
$$

This tensor can be now associated with curve $\gamma_{j}$ only. Furthermore it depends on the external vector $\mathbf{r}_{j}$. One has trivially $\frac{\partial}{\partial \mathbf{r}_{A_{j}}}=\frac{\partial}{\partial \mathbf{r}_{j}}$ and thus

$$
\operatorname{curl}_{A_{j}} d \underset{\sim}{\boldsymbol{\Gamma}}\left(\mathbf{r}_{A_{j} A_{j+1}^{\prime}}+\mathbf{Q}_{j}\right)=\operatorname{curl}_{r_{j}} d \underset{\sim}{\boldsymbol{\Gamma}}\left(\mathbf{r}_{j}-\mathbf{r}_{A_{j}^{\prime} A_{j}}\right) .
$$

Combining Eqs. (2.9), (2.17), (2.20), and (2.24), we can write the integral $\mathscr{I}$ in the form :

$$
\begin{aligned}
\mathscr{I}\left(\gamma_{1}, \ldots, \gamma_{n}\right)= & \int d^{3} r_{1} \ldots d^{3} r_{n} \delta\left(\mathbf{r}_{1}+\ldots+\mathbf{r}_{n}\right) \\
& \cdot \operatorname{tr}\left\{\prod_{j=n}^{1} \int \frac{d \mathcal{O}_{j}}{8 \pi^{2}} \operatorname{curl}_{r_{j}}\left[\oint_{A_{j}} \oint_{A_{j}^{\prime}} d \underset{\sim}{\mathbf{r}_{j}}\left(\mathbf{r}_{j}-\mathbf{r}_{A_{j}^{\prime} A_{j}}\right)\right]\right\},
\end{aligned}
$$

after some permutations of integrations and derivations.

It is now convenient to introduce the characteristic tensor $\boldsymbol{\Gamma}(\mathbf{r})$, associated with a given curve $\gamma$, which has generic points $A, A^{\prime}$ :

$$
\underset{\sim}{\Gamma}(\mathbf{r})=\int \frac{d \mathcal{O}}{8 \pi^{2}} \oint_{A} \oint_{A^{\prime}} d \underset{\sim}{\mathbf{\Gamma}}\left(\mathbf{r}-\mathbf{r}_{A^{\prime} A}\right)
$$

Owing to (2.11) $\Gamma$ reads explicitly ${ }^{3}$

$$
\underset{\sim}{\Gamma}(\mathbf{r})=-\int \frac{d \mathcal{O}}{8 \pi^{2}} \oint_{\gamma} \oint_{\gamma} \frac{1}{4 \pi} \frac{d \mathbf{r}_{A} \otimes d \mathbf{r}_{A^{\prime}}}{\left|\mathbf{r}-\left(\mathbf{r}_{A^{\prime}}-\mathbf{r}_{A}\right)\right|} .
$$

3 The integrand of (2.27) is defined almost everywhere in $\mathbf{r}$, and its divergence on a domain of measure zero are regularized by rotation. Thus $\Gamma$ is defined everywhere in $\mathbf{r}$ 
This tensor is obtained by integration over orientations $\mathcal{O}$ of the curve $\gamma$ and depends only on one external vector $\mathbf{r}$.

Owing to this definition, the integral $\mathscr{I}(2.23)$ takes then the simple form:

$$
\mathscr{I}\left(\gamma_{1}, \ldots, \gamma_{n}\right)=\int d^{3} r_{1} \ldots d^{3} r_{n} \delta\left(\mathbf{r}_{1}+\ldots+\mathbf{r}_{n}\right) \operatorname{tr}\left\{\prod_{j=n}^{1}\left[\operatorname{curl}_{r_{j} \sim j} \underset{j}{\mathbf{r}_{j}}\left(\mathbf{r}_{j}\right)\right]\right\} .
$$

The presence of a convolution integral and of a tensorial trace originates in the ring structure of the $n$ "linked" curves.

\section{The Characteristic Tensor $\Gamma$}

The aim of this section is to calculate the tensor curl $\Gamma$ which appears in (2.28). We shall consider for a given curve $\gamma$, the characteristic tensor $\Gamma$ and show that $\Gamma$, and thus its curl, can be written as the sum of two terms. The latter involve two characteristic functions of curve $\gamma$ and are related to different geometrical properties of $\gamma$.

Consider $\Gamma$ given by (2.27). The divergence of this tensor, acting on the left indices and on $\mathbf{r}$, reads:

$$
\operatorname{div} \underset{\sim}{\Gamma}(\mathbf{r})=-\int \frac{d \mathcal{O}}{8 \pi^{2}} \oint \oint \frac{1}{4 \pi}\left(\nabla_{r} \frac{1}{\left|\mathbf{r}-\mathbf{r}_{A^{\prime} A}\right|} \cdot d \mathbf{r}_{A}\right) d \mathbf{r}_{A^{\prime}} .
$$

Using $\nabla_{r}=\nabla_{A}$, we see that the right hand side involves the circulation of a gradient along closed curve $\gamma$, which therefore vanishes:

$$
\oint \nabla_{A} \frac{1}{\left|\mathbf{r}-\mathbf{r}_{A^{\prime} A}\right|} \cdot d \mathbf{r}_{A}=0 .
$$

Thus $^{4}$

$$
\operatorname{div} \underset{\sim}{\boldsymbol{\Gamma}}(\mathbf{r})=\mathbf{0}
$$

We then have the following lemma:

Lemma. Any tensor $\underset{\sim}{\boldsymbol{\Gamma}}(\mathbf{r})$ of rank two, depending on only one vector $\mathbf{r}$, and divergencefree, can be written

$$
\underset{\sim}{\boldsymbol{\Gamma}}(\mathbf{r})=\operatorname{curl} \operatorname{curl}[\varphi(r) \mathbf{1}]+\operatorname{curl}[\psi(r) \mathbf{1}],
$$

where $\varphi$ and $\psi$ are functions of modulus $r=|\mathbf{r}| \cdot \mathbf{1}$ is the unit tensor.

The curl acts as before on the left indices. The derivatives act evidently on $\mathbf{r}$ and the subscript $r$ has been dropped. This lemma is proved in Appendix A. Incidentally, we note that one operator curl acting on a tensor, changes its symmetry properties with respect to the indices. Two curl operators leave them invariant. Thus, because $\varphi \mathbf{1}$ and $\psi \mathbf{1}$ are symmetric, the first term of the right hand side of (3.3) gives the symmetric part of $\Gamma$, whereas the second term gives the antisymmetric part. We shall relate below the functions $\varphi, \psi$ to the invariant

4 The right divergence also vanishes 
elements of the tensor $\Gamma$. Our interest lies mainly in the expression of curl $\Gamma$, which reads

$$
\operatorname{curl} \Gamma \Gamma=\operatorname{curl} \operatorname{curl} \operatorname{curl}(\varphi \mathbf{1})+\operatorname{curl} \operatorname{curl}(\psi \mathbf{1}) .
$$

Using the identity

$$
\operatorname{curl} \operatorname{curl}=\nabla \operatorname{div}-\nabla^{2},
$$

we get

$$
\begin{aligned}
\underset{\sim}{\operatorname{curl} \Gamma \underset{\Gamma}{\Gamma}} & =\left(\nabla \operatorname{div}-\nabla^{2}\right) \operatorname{curl}(\varphi \mathbf{1})+\operatorname{curl} \operatorname{curl}(\psi \mathbf{1}) \\
& =-\operatorname{curl}\left(\nabla^{2} \varphi \mathbf{1}\right)+\operatorname{curl} \operatorname{curl}(\psi \mathbf{1}),
\end{aligned}
$$

where we have used div curl $=0$. The first term of the right hand side of (3.6) gives the antisymmetric part of curl $\underset{\sim}{\Gamma}$, the second one being symmetric. We note now that for any function $f(r)$ :

$$
\operatorname{div}(f \mathbf{1})=\nabla f .
$$

This, together with (3.5), gives the useful identity

$$
\operatorname{curl} \operatorname{curl}(f \mathbf{1})=\left(\nabla \otimes \nabla-1 \nabla^{2}\right) f .
$$

On the other hand, one has trivially

$$
\operatorname{curl}(f \mathbf{1})=\nabla f \wedge \mathbf{1} .
$$

Taking the trace of these identities, we get

$$
\begin{aligned}
\operatorname{tr} \operatorname{curl} \operatorname{curl}(f \mathbf{1}) & =\left[\nabla^{2}-(\operatorname{tr} \mathbf{1}) \nabla^{2}\right] f \\
& =-2 \nabla^{2} f
\end{aligned}
$$

and

$$
\operatorname{tr} \operatorname{curl}(f \mathbf{1})=0 .
$$

As the tensor $\operatorname{curl}(f \mathbf{1})$ is antisymmetric this result was expected and we have also, for the same reason:

$$
\operatorname{tr} \operatorname{curl} \operatorname{curl} \operatorname{curl}(f \mathbf{1})=0 .
$$

Applying (3.10) and (3.11a, b) to the expressions (3.3) and (3.4) of $\underset{\sim}{\Gamma}$ and curl $\underset{\sim}{\Gamma}$, we immediately get

$$
\begin{aligned}
\operatorname{tr} \underset{\sim}{\Gamma}(\mathbf{r}) & =-2 \nabla^{2} \varphi(r) \\
\operatorname{tr} \operatorname{curl} \underset{\sim}{\Gamma}(\mathbf{r}) & =-2 \nabla^{2} \psi(r) .
\end{aligned}
$$

Thus $\varphi, \psi$ are given by two Laplace equations, of which the sources are traces of $\Gamma$ and its curl. Let us summarize these results by giving the useful form of curl $\underset{\sim}{\Gamma}$ [use (3.6), (3.8), (3.9), and (3.12)]:

$$
\operatorname{curl} \underset{\sim}{\Gamma}=\frac{1}{2} \nabla(\operatorname{tr} \underset{\sim}{\Gamma}) \wedge \mathbf{1}+\left(\nabla \otimes \nabla-1 \nabla^{2}\right) \psi,
$$

where $\psi$ is given by Eq. (3.13). 


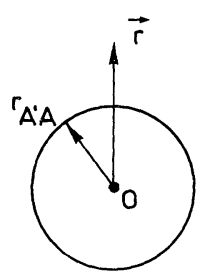

(a)

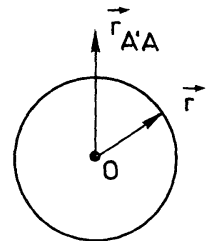

(b)

Fig. 4a and b. Source spheres: a The extremity of $\mathbf{r}_{A^{\prime} A}$ describes the whole sphere of radius $r_{A^{\prime} A}$ when curve $\gamma$ rotates. The Newtonian field is created at $\mathbf{r}$ and vanishes inside the sphere. $\mathbf{b}$ The complementary situation: the extremity of $\mathbf{r}$ describes a sphere of radius $r$ and the Newtonian field is created at $\mathbf{r}_{A^{\prime} A}$

For determining curl $\Gamma$, we now calculate the vector $\nabla \operatorname{tr} \underset{\sim}{\Gamma}$ and the invariant $\operatorname{tr} \operatorname{curl} \underset{\sim}{\Gamma}$, which is the source of $\psi$. For that purpose we come back to the definition (2.27) of $\underset{\sim}{\Gamma}$ and get immediately

$$
\begin{gathered}
\nabla \operatorname{tr} \underset{\sim}{\Gamma}(\mathbf{r})=-\int \frac{d \Theta}{8 \pi^{2}} \oint \oint d \mathbf{r}_{A} \cdot d \mathbf{r}_{A^{\prime}} \frac{1}{4 \pi} \nabla_{r} \frac{1}{\left|\mathbf{r}-\mathbf{r}_{A^{\prime} A}\right|} \\
\operatorname{tr} \operatorname{curl} \underset{\sim}{\Gamma}(\mathbf{r})=-\int \frac{d \Theta}{8 \pi^{2}} \oint \oint \frac{1}{4 \pi}\left(\nabla_{r} \frac{1}{\left|\mathbf{r}-\mathbf{r}_{A^{\prime} A}\right|}, d \mathbf{r}_{A}, d \mathbf{r}_{A^{\prime}}\right),
\end{gathered}
$$

where $A, A^{\prime}$ are two generic points on curve $\gamma$.

In the first quantity (3.15) the differential term $d \mathbf{r}_{A} \cdot d \mathbf{r}_{A^{\prime}}$ is invariant under the rotations of the curve $\gamma$ and we can write

$$
\nabla \operatorname{tr} \underset{\sim}{\Gamma}(\mathbf{r})=\frac{1}{4 \pi} \oint \oint d \mathbf{r}_{A} \cdot d \mathbf{r}_{A^{\prime}}\left(-\nabla_{r} \int \frac{d \mathcal{O}}{8 \pi^{2}} \frac{1}{\left|\mathbf{r}-\mathbf{r}_{A^{\prime} A}\right|}\right) .
$$

The following vectorial function therefore appears :

$$
-\nabla_{r} \int \frac{d \Theta}{8 \pi^{2}} \frac{1}{\left|\mathbf{r}-\mathbf{r}_{A^{\prime} A}\right|}
$$

$\frac{1}{\left|\mathbf{r}-\mathbf{r}_{A^{\prime} A}\right|}$ is the Newtonian potential created at $\mathbf{r}$ by a source located at $\mathbf{r}_{A^{\prime} A}$ (Fig. 4a). When curve $\gamma$ rotates in space, vector $\mathbf{r}_{A^{\prime} A}$, which is attached to $\gamma$, takes any orientation in space. With respect to a fixed origin 0 the extremity of vector $\mathbf{r}_{A^{\prime} A}$ describes a sphere of radius $r_{A^{\prime} A}$. Therefore (3.18) is a Newtonian field created by a spherical distribution of masses. Applying Gauss' theorem [2], we can write directly

$$
\nabla_{r} \int \frac{d \mathcal{O}}{8 \pi^{2}} \frac{1}{\left|\mathbf{r}-\mathbf{r}_{A^{\prime} A}\right|}=\theta\left(r-r_{A^{\prime} A}\right) \nabla\left(\frac{1}{r}\right) .
$$

The $\theta$-distribution accounts for the vanishing of a Newtonian field inside its source sphere. Thus we obtain for vector (3.15) the simple expression:

$$
\nabla \operatorname{tr} \underset{\sim}{\Gamma}(\mathbf{r})=-\frac{1}{4 \pi} \nabla\left(\frac{1}{r}\right) \oint \oint d \mathbf{r}_{A} \cdot d \mathbf{r}_{A^{\prime}} \theta\left(r-r_{A^{\prime} A}\right) .
$$


Let us now consider the expression (3.16). Permuting the curvilinear integrals and the integral over orientations, we first calculate the infinitesimal quantity

$$
\int \frac{d \Theta}{8 \pi^{2}}\left(\nabla_{r} \frac{1}{\left|\mathbf{r}-\mathbf{r}_{A^{\prime} A}\right|}, d \mathbf{r}_{A}, d \mathbf{r}_{A^{\prime}}\right)
$$

The determinant is a scalar number depending on the orientation of two independent solid bodies, which are curve $\gamma$ and vector $\mathbf{r}$. Thus (3.21) is also equal to its own average over orientations of unit vector $\hat{r}=\mathbf{r} / r$, given by:

$$
\left(\nabla_{A} \int \frac{d^{2} \hat{r}}{4 \pi} \frac{1}{\left|\mathbf{r}-\mathbf{r}_{A^{\prime} A}\right|}, d \mathbf{r}_{A}, d \mathbf{r}_{A^{\prime}}\right)
$$

where $\nabla_{r}=\nabla_{A}$. Now $\mathbf{r}$ is the source point of the Newtonian field and describes the whole sphere of radius $r$. Gauss' theorem yields directly

$$
\nabla_{A} \int \frac{d^{2} \hat{r}}{4 \pi} \frac{1}{\left|\mathbf{r}-\mathbf{r}_{A^{\prime} A}\right|}=\theta\left(r_{A^{\prime} A}-r\right) \nabla_{A}\left(\frac{1}{r_{A^{\prime} A}}\right)
$$

We finally obtain a simple expression of trace (3.21):

$$
\operatorname{tr} \operatorname{curl} \underset{\sim}{\Gamma}(\mathbf{r})=-\frac{1}{4 \pi} \oint \oint\left(\nabla_{A} \frac{1}{r_{A^{\prime} A}}, d \mathbf{r}_{A}, d \mathbf{r}_{A^{\prime}}\right) \theta\left(r_{A^{\prime} A}-r\right) .
$$

It will be convenient, in order to relate to results of $[3,4]$, to rewrite Eqs. $(3.20)$ and (3.23) in the form:

$$
\begin{aligned}
\nabla \operatorname{tr} \underset{\sim}{\Gamma}(r) & =\frac{\mathbf{r}}{r^{2}} \mathscr{A}(r), \\
\operatorname{tr} \operatorname{curl} \prod_{\sim}(r) & =\frac{1}{r} \mathscr{B}(r) .
\end{aligned}
$$

$\mathscr{A}, \mathscr{B}$ are two characteristic functions associated with a given curve:

$$
\begin{aligned}
& \mathscr{A}(r)=\frac{1}{4 \pi r} \oint \oint d \mathbf{r}_{A} \cdot d \mathbf{r}_{A^{\prime}} \theta\left(r-r_{A A^{\prime}}\right), \\
& \mathscr{B}(r)=-\frac{r}{4 \pi} \oint \oint\left(\nabla_{A} \frac{1}{r_{A A^{\prime}}}, d \mathbf{r}_{A}, d \mathbf{r}_{A^{\prime}}\right) \theta\left(r_{A A^{\prime}}-r\right) .
\end{aligned}
$$

$\mathscr{A}$ is, up to a normalization factor, the function introduced by Pohl [3] for plane curves. The presence of a second characteristic function, besides $\mathscr{A}$, was obtained in [4], but a different function $\mathscr{B}$ was introduced.

Our aim was to calculate the tensor curl $\Gamma$ which appears in Eq. (2.28) and is the fundamental object of our linking study. Using its expressions (3.14) and (3.24) we get in terms of $\mathscr{A}, \mathscr{B}$ :

$$
\operatorname{curl} \underset{\sim}{\Gamma}(\mathbf{r})=\frac{1}{2}(\mathbf{r} \wedge \mathbf{1}) \frac{1}{r^{2}} \mathscr{A}(r)+\left(\nabla \otimes \nabla-1 \nabla^{2}\right) \psi(r)
$$


where $\psi$ satisfies the Laplace equation

$$
\nabla^{2} \psi(r)=-\frac{1}{2 r} \mathscr{B}(r)
$$

obtained from (3.13) and (3.24).

\section{The Kinematic Linking Integral for Two Curves}

We shall evaluate in this section the integral $\mathscr{I}$ for two curves $\gamma_{1}, \gamma_{2}$. Their characteristic tensors are respectively $\Gamma_{1}$ and $\Gamma_{2}$ and the associated characteristic functions are $\mathscr{A}_{1}, \mathscr{B}_{1}$ and $\mathscr{A}_{2}, \mathscr{B}_{2}$. We shall prove the generalization of Pohl's theorem [1], given in [2]:

The integral of the squared number $I^{2}$ of two curves, over their random rigid motions can be factorized in a single integral

$$
\begin{aligned}
\frac{1}{8 \pi^{2}} \int d^{3} \varrho d O I^{2} & =\mathscr{I}\left(\gamma_{1}, \gamma_{2}\right) \\
& =2 \pi \int_{0}^{\infty} d r\left[\mathscr{A}_{1}(r) \mathscr{A}_{2}(r)+\mathscr{B}_{1}(r) \mathscr{B}_{2}(r)\right] .
\end{aligned}
$$

To prove this formula, we have to calculate, owing to (2.28):

$$
\begin{aligned}
\mathscr{I}\left(\gamma_{1}, \gamma_{2}\right) & =\int d^{3} r_{1} d^{3} r_{2} \delta\left(\mathbf{r}_{1}+\mathbf{r}_{2}\right) \operatorname{tr}\left[\operatorname{curl} \underset{\sim}{\Gamma_{2}}\left(\mathbf{r}_{2}\right) \operatorname{curl}{\underset{\sim}{\Gamma}}_{1}\left(\mathbf{r}_{1}\right)\right] \\
& =\int d^{3} r \operatorname{tr}\left[\operatorname{curl} \underset{\sim}{\Gamma_{2}}(r) \operatorname{curl}{\underset{\sim}{\mid}}_{1}(-\mathbf{r})\right] .
\end{aligned}
$$

In trace tr, symmetric and antisymmetric parts of curl $\Gamma_{1}$ and curl $\Gamma_{2}$ decouple completely. According to Eq. (3.27), we thus find:

$$
\begin{aligned}
\mathscr{I}\left(\gamma_{1}, \gamma_{2}\right)= & \int d^{3} r \operatorname{tr}[-(\mathbf{r} \wedge \mathbf{1})(\mathbf{r} \wedge \mathbf{1})] \frac{1}{4 r^{4}} \mathscr{A}_{1}(r) \mathscr{A}_{2}(r) \\
& +\int d^{3} r \operatorname{tr}\left\{\left[\left(\nabla \otimes \nabla-\mathbf{1} \nabla^{2}\right) \psi_{1}(r)\right]\left[\left(\boldsymbol{\nabla} \otimes \nabla-1 \nabla^{2}\right) \psi_{2}(r)\right]\right\} .
\end{aligned}
$$

Using

$$
(\mathbf{r} \wedge \mathbf{1})(\mathbf{r} \wedge \mathbf{1})=\mathbf{r} \wedge(\mathbf{r} \wedge \mathbf{1})=\mathbf{r} \otimes \mathbf{r}-r^{2} \mathbf{1}
$$

we get

$$
\operatorname{tr}[-(\mathbf{r} \wedge \mathbf{1})(\mathbf{r} \wedge \mathbf{1})]=2 r^{2}
$$

On the other hand, the second term in the right hand side of Eq. (4.1) can be written by expanding the product of tensors and using different notations

$$
\int d^{3} r\left\{\operatorname{tr}\left[\left(\nabla \psi_{1}(r) \nabla\right)\left(\nabla \psi_{2}(r) \nabla\right)\right]+\nabla^{2} \psi_{1}(r) \nabla^{2} \psi_{2}(r)\right\}
$$

Integrating by parts the first term gives twice the product of Laplacians

$$
\int d^{3} r 2 \nabla^{2} \psi_{1}(r) \nabla^{2} \psi_{2}(r)=2 \int d^{3} r \frac{1}{4 r^{2}} \mathscr{B}_{1}(r) \mathscr{B}_{2}(r),
$$




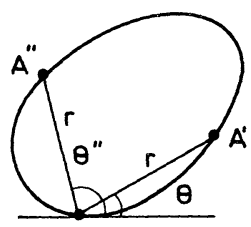

A

Fig. 5. Points $A^{\prime}$ and $A^{\prime \prime}$ contributing to function $\mathscr{A}(r)$

where we have made use in the end of Laplace's equation (3.28). Gathering the preceding formulae and performing in (4.2) a trivial angular integration, we get finally the announced result:

$$
\mathscr{I}\left(\gamma_{1}, \gamma_{2}\right)=2 \pi \int_{0}^{\infty} d r\left[\mathscr{A}_{1}(r) \mathscr{A}_{2}(r)+\mathscr{B}_{1}(r) \mathscr{B}_{2}(r)\right] .
$$

Let us recall the form of associated functions of a given curve $\gamma$ :

$$
\begin{aligned}
& \mathscr{A}(r)=\frac{1}{4 \pi r} \oint_{\gamma} \oint_{\gamma} d \mathbf{r}_{A} \cdot d \mathbf{r}_{A^{\prime}} \theta\left(r-r_{A A^{\prime}}\right), \\
& \mathscr{B}(r)=-\frac{r}{4 \pi} \oint_{\gamma} \oint_{\gamma}\left(\nabla_{A} \frac{1}{r_{A A^{\prime}}}, d \mathbf{r}_{A}, d \mathbf{r}_{A^{\prime}}\right) \theta\left(r_{A A^{\prime}}-r\right) .
\end{aligned}
$$

$\mathscr{B}$ contains the determinant of three vectors, which are coplanar if the curve itself is plane. Thus, for a plane curve, $\mathscr{B}$ vanishes identically. Then (4.2) reduces to the result (1.4) established by Pohl [3], by quite a different method, for two plane and convex curves. For twisted curves, a result similar to (4.2) has been obtained by des Cloizeaux and Ball [4]. As they used a different method, they found in fact a different function $\mathscr{B}$. The form of $\mathscr{A}$ and $\mathscr{B}$ is not unique for expressing the integral $\mathscr{I}$ for two curves. We show in Appendix B the equivalence between (4.2) and the result of [4]. Let us illustrate Theorem (4.2) by recalling some properties of characteristic functions $\mathscr{A}, \mathscr{B}$.

Consider function $\mathscr{A}$ (4.3a). It can be transformed easily (see [4] for details), by integrating by parts, into:

$$
\mathscr{A}(r)=\frac{1}{4 \pi} \oint \oint\left(d \mathbf{r}_{A} \cdot \hat{r}_{A A^{\prime}}\right)\left(d \mathbf{r}_{A^{\prime}} \cdot \hat{r}_{A A^{\prime}}\right) \delta\left(r-r_{A A^{\prime}}\right) .
$$

In this formula, points, $A, A^{\prime}$ on the curve are distant of $r$. Integrating the distribution gives the original form of Pohl's result [3]:

$$
\mathscr{A}(r)=\frac{1}{4 \pi} \int_{\gamma} d s|\cos \theta| \text {. }
$$

$s$ is the curvilinear abscissa of point $A$ along the curve. The angle $\theta$ is formed by the tangent vector at $A$ and the vector $A A^{\prime}$ ( $A^{\prime}$ being at a distance $r$ of $A$ ) (Fig. 5). The closed curve $\gamma$ has been implicitly assumed to have a tangent at each point. It can thus be rectified. For $r=0$ :

$$
\mathscr{A}(0)=\frac{L}{2 \pi}
$$


where $L$ is the length of the curve. A factor 2 is present, because, for a smooth curve having a tangent at each point, there are two points $A^{\prime}$, say $A_{+}, A_{-}$, infinitesimally close to point $A$, lying on either side of it.

Consider now the second characteristic function $\mathscr{B}$, given by (4.3b). It involves a determinant, the sign of which depends on space orientation. Thus, if $\gamma^{\prime}$ is the image of a curve $\gamma$ by symmetry (with respect to a point or a plane), its characteristic function $\mathscr{B}_{\gamma^{\prime}}$ is equal to

$$
\mathscr{B}_{\gamma^{\prime}}=-\mathscr{B}_{\gamma} .
$$

Any curve symmetric with respect to a point or a plane possesses a vanishing $\mathscr{B}$ function. Such is the case of plane curves.

When $r$ approaches 0 :

$$
\mathscr{B}(r) \simeq r I_{\text {self }} \quad(r \rightarrow 0),
$$

where the real number $I_{\text {self }}$ is given by

$$
I_{\mathrm{selv}}=-\frac{1}{4 \pi} \oint_{\gamma} \oint_{\gamma}\left(\nabla_{A} \frac{1}{r_{A A^{\prime}}}, d \mathbf{r}_{A}, d \mathbf{r}_{A^{\prime}}\right) .
$$

This is the formal Gauss linking number of curve $\gamma$ superposed to itself ${ }^{5}$. Let us finally note that $\mathscr{A}$ and $\mathscr{B}$ have compact supports. Equations (4.4) and (4.3b) clearly show indeed that:

$$
\left.\begin{array}{l}
\mathscr{A}(r)=0 \\
\mathscr{B}(r)=0
\end{array}\right\} \forall r>\phi(\gamma),
$$

where $\phi(\gamma)$ is $\gamma$ 's diameter.

\section{The Kinematic Linking Integral of an Arbitrary Number of Curves}

The aim of this section is to present a new factorization theorem for the kinematic integral $\mathscr{I}$ of the linkings of $n$ curves. We show in particular that this factorization involves special algebraic rules.

We want to evaluate the convolution integral $\mathscr{I}$ (2.28), and, for $n$ greater than 2, the simplest way is to use Fourier transforms. We then define the Fourier transform $\operatorname{curl} \Gamma(\mathbf{p})$ of tensor $\operatorname{curl} \Gamma(\mathbf{r})$ by:

$$
\widehat{\operatorname{curl}} \underset{\sim}{\Gamma}(\mathbf{p})=\int d^{3} r e^{i \mathbf{p} \cdot \mathbf{r}} \operatorname{curl} \underset{\sim}{\Gamma}(\mathbf{r}) \text {. }
$$

Each curve $\gamma_{j}$ possesses such a tensor. Using the Fourier representation

$$
\delta\left(\mathbf{r}_{1}+\ldots+\mathbf{r}_{n}\right)=\frac{1}{(2 \pi)^{3}} \int d^{3} p e^{i \mathbf{p} \cdot\left(\mathbf{r}_{1}+\ldots+\mathbf{r}_{n}\right)},
$$

and the Eq. (5.1), we trivially obtain:

$$
\mathscr{I}\left(\gamma_{1}, \ldots, \gamma_{n}\right)=\frac{1}{(2 \pi)^{3}} \int d^{3} p \operatorname{tr}\left[{\widehat{\operatorname{curl}} \underset{\sim}{\Gamma}}_{n}(\mathbf{p}) \ldots \widehat{\operatorname{curl} \Gamma_{1}}(\mathbf{p})\right]
$$

5 It is not however an invariant counting the number of knots of $\gamma$ 
We can obtain $\operatorname{curl} \Gamma$ from Eqs. (3.27) and (3.28): we need to define the Fourier transforms

$$
\begin{aligned}
& \underset{\sim}{\mathbf{A}}(\mathbf{p})=\int d^{3} r e^{i \mathbf{p} \cdot \mathbf{r}}(\mathbf{r} \wedge \mathbf{1}) \frac{1}{2 r^{2}} \mathscr{A}(r) \\
& \underset{\sim}{\mathbf{B}}(\mathbf{p})=-\int d^{3} r e^{i \mathbf{p} \cdot \mathbf{r}}(\nabla \otimes \nabla-\mathbf{1} \Delta) \Delta^{-1}\left[\frac{\mathscr{B}(r)}{2 r}\right],
\end{aligned}
$$

where we conventionally call $\Delta^{-1}$ the solution $\psi$ of the Laplace equation (3.28). We have

$$
\widehat{\operatorname{curl}} \underset{\sim}{\Gamma}(\mathbf{p})=\underset{\sim}{\mathbf{A}}(\mathbf{p})+\underset{\sim}{\mathbf{B}}(\mathbf{p}) .
$$

By using the identity

$$
\int d^{2} \hat{r} e^{i \mathbf{p} \cdot \mathbf{r}}=4 \pi \frac{\sin p r}{p r},
$$

one finds easily, after some manipulations, the Fourier transforms:

$$
\begin{aligned}
& \underset{\sim}{\mathbf{A}}(\mathbf{p})=i(\hat{p} \wedge \mathbf{1}) \mathscr{A}[p), \\
& \underset{\sim}{\mathbf{B}}(\mathbf{p})=(\mathbf{1}-\hat{p} \otimes \hat{p}) \mathscr{B}[p]
\end{aligned}
$$

$(\hat{p}=\mathbf{p} / p)$, where $\mathscr{A}[p], \mathscr{B}[p]$ are scalar Fourier transforms, defined by:

$$
\begin{aligned}
& \mathscr{A}[p]=-2 \pi \frac{d}{d p} \int_{0}^{\infty} d r \frac{\sin p r}{p r} \mathscr{A}(r) \\
& \mathscr{B}[p]=2 \pi \frac{1}{p} \int_{0}^{\infty} d r \sin \operatorname{pr} \mathscr{B}(r) .
\end{aligned}
$$

Therefore $\operatorname{curl} \Gamma$ finally reads:

$$
\widehat{\operatorname{curl}} \underset{\sim}{\Gamma}(\mathbf{p})=i(\hat{p} \wedge \mathbf{1}) \mathscr{A}[p]+(\mathbf{1}-\hat{p} \otimes \hat{p}) \mathscr{B}[p] .
$$

Because $\mathscr{A}(r)$ and $\mathscr{B}(r)$ have compact supports in "real" space, the associated functions $\mathscr{A}[p], \mathscr{B}[p]$ always exist. The explicit expressions of the characteristic functions $\mathscr{A}[p], \mathscr{B}[p]$ in momentum space are given in Appendix $\mathrm{C}$.

The integral $\mathscr{I}(5.3)$ reads therefore

$$
\mathscr{I}\left(\gamma_{1}, \ldots, \gamma_{n}\right)=\frac{1}{(2 \pi)^{3}} \int d^{3} p \operatorname{tr} \underset{\sim}{\mathbf{P}}(\mathbf{p})
$$

where $\underset{\sim}{\mathbf{P}}(\mathbf{p})$ is the following product of matrices :

$$
\left.\underset{\sim}{\mathbf{P}}(\mathbf{p})=\prod_{j=n}^{1} \widehat{\operatorname{curl} \Gamma_{j}}(\mathbf{p})\right]=\prod_{j=n}^{1}\left[i(\hat{p} \wedge \mathbf{1}) \mathscr{A}_{j}[p]+(\mathbf{1}-\hat{p} \otimes \hat{p}) \mathscr{B}_{j}[p]\right] .
$$

For evaluating this product, it suffices to calculate four partial products:

$$
\begin{aligned}
i(\hat{p} \wedge \mathbf{1}) i(\hat{p} \wedge \mathbf{1}) & =\mathbf{1}-\hat{p} \otimes \hat{p}, \\
i(\hat{p} \wedge \mathbf{1})(\mathbf{1}-\hat{p} \otimes \hat{p}) & =i \hat{p} \wedge 1, \\
(\mathbf{1}-\hat{p} \otimes \hat{p}) i(\hat{p} \wedge \mathbf{1}) & =i \hat{p} \wedge \mathbf{1}, \\
(\mathbf{1}-\hat{p} \otimes \hat{p})(\mathbf{1}-\hat{p} \otimes \hat{p}) & =\mathbf{1}-\hat{p} \otimes \hat{p} .
\end{aligned}
$$


Thus the algebra of operators $i \hat{p} \wedge \mathbf{1}$ and $\mathbf{1}-\hat{p} \otimes \hat{p}$ is trivially isomorphic with a set of two objects $\varepsilon, 1$ :

$$
\begin{array}{r}
i \hat{p} \wedge \mathbf{1} \rightarrow \varepsilon, \\
\mathbf{1}-\hat{p} \otimes \hat{p} \rightarrow 1,
\end{array}
$$

obeying the algebraic rules:

$$
\varepsilon^{2}=1, \quad 1 \cdot \varepsilon=\varepsilon \cdot 1=\varepsilon, \quad 1^{2}=1 .
$$

The image of tensor $\underset{\sim}{\mathbf{P}}$, given by this isomorphism is :

$$
\mathscr{P}[p]=\prod_{j=1}^{n}\left(\varepsilon \mathscr{A}_{j}[p]+\mathscr{B}_{j}[p]\right) .
$$

According to rules (5.16), $\mathscr{P}$ can always be written as a linear combination of 1 and $\varepsilon$ :

$$
\mathscr{P}[p]=\mathscr{X}[p]+\varepsilon \mathscr{Y}[p] .
$$

Tensor $\underset{\sim}{\mathbf{P}}$ then takes the form:

$$
\underset{\sim}{\mathbf{P}}(\mathbf{p})=(\mathbf{1}-\hat{p} \otimes \hat{p}) \mathscr{X}[p]+i(\hat{p} \wedge \mathbf{1}) \mathscr{Y}[p] .
$$

Calculating the traces

$$
\operatorname{tr}(\mathbf{1}-\hat{p} \otimes \hat{p})=2, \quad \operatorname{tr}(\hat{p} \wedge \mathbf{1})=0,
$$

we obtain:

$$
\operatorname{tr} \underset{\sim}{\mathbf{P}}(\mathbf{p})=2 \mathscr{X}[p] \text {. }
$$

Then integral (5.12) can be partially performed on angular variables, and this finally leads us to the following factorization theorem:

Theorem. The integral $\mathscr{I}$ of the cyclic product of the linking numbers of a set of $n$ curves $\gamma_{j}$, over random rigid motions of the latter, can be factorized into the single integral:

$$
\mathscr{I}\left(\gamma_{1}, \ldots, \gamma_{n}\right)=\frac{1}{\pi^{2}} \int_{0}^{\infty} d p p^{2} \mathscr{X}[p]
$$

where function $\mathscr{X}[p]$ is the even part of the algebraic product:

$$
\begin{aligned}
\mathscr{P}[p] & =\prod_{j=1}^{n}\left(\varepsilon \mathscr{A}_{j}[p]+\mathscr{B}_{j}[p]\right) \quad\left(\varepsilon^{2}=1\right) \\
& =\mathscr{X}[p]+\varepsilon \mathscr{Y}[p] .
\end{aligned}
$$

Many properties of integral $\mathscr{I}$ appear immediately in this result.

For two curves $\gamma_{1}$ and $\gamma_{2}$, the even and odd parts $\mathscr{X}$ and $\mathscr{Y}$ read respectively:

$$
\begin{aligned}
& \mathscr{X}=\mathscr{A}_{1} \mathscr{A}_{2}+\mathscr{B}_{1} \mathscr{B}_{2}, \\
& \mathscr{Y}=\mathscr{A}_{1} \mathscr{B}_{2}+\mathscr{B}_{1} \mathscr{A}_{2} .
\end{aligned}
$$

One recognizes in $\mathscr{X}$, the expression, in momentum space, of the usual Eq. (4.2) for two curves.

One must also note that the product $\mathscr{P}(5.22 \mathrm{~b})$ is abelian. 


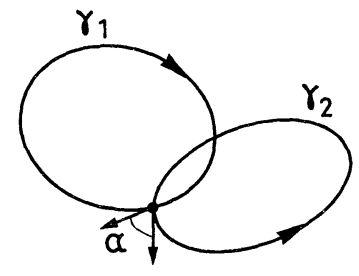

Fig. 6. A contact point between curves $\gamma_{1}, \gamma_{2}$

Corollary. The kinematic integral $\mathscr{I}$ of cyclic linkings of an ordered set of $n$ curves, arranged in a ring, is in fact independent of the order of the curves.

If curves $\gamma_{j}$ are plane, all characteristic functions $\mathscr{B}_{j}$ vanish. Then

$$
\mathscr{P}[p]=\varepsilon^{n} \prod_{j=1}^{n} \mathscr{A}_{j}[p] .
$$

Therefore we find that real part $\mathscr{X}$ vanishes for $n$ odd, and so does integral $\mathscr{I}$. Indeed one notes that a symmetry of two curves $j, j+1$ with respect to a plane, changes their algebraic linking number into

$$
I_{j j+1}^{\prime}=-I_{j j+1}
$$

Thus, for $n$ curves, the symmetry gives a factor $(-1)^{n}$. Plane curves can always be superposed to their symmetric images by motions in space. In this case, a direct configuration and its image will both contribute to integral $\mathscr{I}$, and will cancel each other if $n$ is odd.

By definition, integral $\mathscr{I}$ is convergent. This can be checked on integral (5.22a), by using the asymptotic behaviours of $\mathscr{A}[p], \mathscr{B}[p]$ for $p \rightarrow 0$ and $p \rightarrow \infty$, which are given in Appendix C.

\section{Contact Functions and Mutual Inductances of a Random Set of Curves}

The $\mathscr{Y}$ term of expansion (5.22b) should be interpreted. The algebra of the characteristic functions $\mathscr{A}$ and $\mathscr{B}$ can be associated, apart from linkings, to other geometrical properties of curves, in particular contacts and mutual inductions.

For two curves $\gamma_{1}$ and $\gamma_{2}$ we define two quantities $C$ and $M$ :

$$
\begin{gathered}
C_{12}=\oint_{\gamma_{1}} \oint_{\gamma_{2}} d \mathbf{r}_{1} \cdot d \mathbf{r}_{2} \delta\left(\mathbf{r}_{12}\right) \\
M_{12}=\int_{\gamma_{1}} \oint_{\gamma_{2}} \frac{1}{4 \pi} \frac{d \mathbf{r}_{1} \cdot d \mathbf{r}_{2}}{\left|\mathbf{r}_{12}\right|},
\end{gathered}
$$

where $\mathbf{r}_{1}, \mathbf{r}_{2}$ belong to curves $\gamma_{1}$ and $\gamma_{2}$ respectively, and where $\mathbf{r}_{12}=\mathbf{r}_{1}-\mathbf{r}_{2}$.

Quantity $C$ measures contacts between oriented curves. It can be written

$$
C_{12}=\int d s_{1} \int d s_{2} \cos \alpha \delta\left(\mathbf{r}_{1}\left(s_{1}\right)-\mathbf{r}_{2}\left(s_{2}\right)\right),
$$

$s_{1}$ and $s_{2}$ are curvilinear coordinates along curves $\gamma_{1}$ and $\gamma_{2}$, and $\alpha$ is the angle formed by the unit tangent vectors at the contact point of the curves (Fig. 6). 
The second quantity $M$ appears in electromagnetism. It is the mutual inductance, also called Neumann's potential [5], and represents the interaction energy between two curves, along which circulate two unit currents.

Now consider the product (2.6) $\prod_{j=1}^{n} I_{j j+1}$. Suppose that a factor $I_{k k+1}$, given by (2.1), is replaced by mutual inductance $M_{k k+1}$ of curves $\gamma_{k}, \gamma_{k+1}$,

$$
M_{k k+1}=\frac{1}{4 \pi} \oint_{\gamma_{k}} \oint_{\gamma_{k+1}} \frac{d \mathbf{r}_{A_{k}} \cdot d \mathbf{r}_{A_{k+1}^{\prime}}}{\left|\mathbf{r}_{A_{k}^{\prime} A_{k+1}}\right|},
$$

likewise a factor $I_{\ell \ell+1}$ is replaced by the contact function $C_{\ell \ell+1}$ of curves $\gamma_{\ell}, \gamma_{\ell+1}$ :

$$
C_{\ell \ell+1}=\oint_{\gamma_{\ell} \gamma_{\ell+1}} \oint_{A_{\ell}} \cdot d \mathbf{r}_{A_{t+1}^{\prime}} \delta\left(\mathbf{r}_{A_{\ell} A_{\ell+1}^{\prime}}\right) \text {. }
$$

Instead of $\prod_{j=1}^{n} I_{j j+1}$, we thus write a cyclic product:

$$
\left[\ldots I_{j j+1} \ldots M_{k k+1} \ldots C_{\ell \ell+1} \ldots\right] .
$$

There are $n$ factors $I, M$ or $C$. The indices go from couple $(1,2)$ for curves $\gamma_{1}, \gamma_{2}$, to $(n, 1)$ for curves $\gamma_{n}, \gamma_{1}$. To calculate product $\prod_{j=1}^{n} I_{j j+1}$, written as a trace of a matrix [see Eqs. (2.14) and (2.15)], we have introduced a set of differential tensors curl $d \Gamma$ (2.13). Each factor $I_{j j+1}$ has generated a term $\operatorname{curl}_{A_{j}} d \underset{\sim}{\boldsymbol{\Gamma}}$ in Eq. (2.15). One can generalize this transformation for product (6.5). We shall find that a factor $M_{k k+1}$ (6.4a) will generate a tensor $-d \boldsymbol{\Gamma}_{k}$ given by Eq. (2.12). Likewise a factor $C_{\ell \ell+1}$, given by $(6.4 \mathrm{~b})$, will generate a tensor $\Delta_{A_{\ell}} d \underset{\sim}{\Gamma_{\ell}}$. The latter is indeed given by the formula $[\operatorname{see}(2.12)]$ :

$$
\Delta_{A_{\ell}} d \boldsymbol{\Gamma}_{\ell}\left(\mathbf{r}_{A_{\ell} A_{\ell+1}}\right)=d \mathbf{r}_{A_{\ell}} \otimes d \mathbf{r}_{A_{\ell}^{\prime}} \delta\left(\mathbf{r}_{A_{\ell} A_{\ell+1}^{\prime}}\right),
$$

and this term, placed in a matricial product, will contribute to build $C_{\ell \ell+1}$. The generalization of Eq. (2.15) can be written

$$
\begin{aligned}
& {\left[\ldots I_{j j+1} \ldots M_{k k+1} \ldots C_{\ell \ell+1} \ldots\right]} \\
& \quad=\ldots \oint_{\gamma_{\ell}} \oint_{\gamma_{\ell}} \ldots \oint_{\gamma_{k}} \oint_{\gamma_{k}} \ldots \oint_{\gamma_{J}} \oint_{\gamma_{J}} \ldots \operatorname{tr}[\ldots \Delta d \underset{\sim}{\boldsymbol{\Gamma}} \ldots(-d \underset{\sim}{\boldsymbol{\Gamma}}) \ldots \operatorname{curl} d \underset{\sim}{\boldsymbol{\Gamma}} \ldots] .
\end{aligned}
$$

As before, the order of curves' indices $j, k, \ell$ is reversed in the matricial product. We now consider the integral of product (6.5) over all relative displacements of curves $\gamma_{j} \cdot j=1, \ldots, n$. The following symbolic notation is used:

$$
\begin{aligned}
& \int_{\text {motions }}\left[\ldots I_{j j+1} \ldots M_{k k+1} \ldots C_{\ell \ell+1} \ldots\right] \\
& \quad=\mathscr{I}\left(\ldots \gamma_{j}, \gamma_{j+1} \ldots \gamma_{k} \sim \gamma_{k+1} \ldots \gamma_{\ell} \cdot \gamma_{\ell+1} \ldots\right) .
\end{aligned}
$$

By convention:

$\gamma_{j}, \gamma_{j+1}$ corresponds to linking number $I_{j j+1}$ between curves $\gamma_{j}$ and $\gamma_{j+1}$.

$\gamma_{k} \sim \gamma_{k+1}$ corresponds to mutual inductance $M_{k k+1}$ between curves $\gamma_{k}$ and $\gamma_{k+1}$. $\gamma_{\ell} \cdot \gamma_{\ell+1}$ corresponds to contact function $C_{\ell \ell+1}$ between curves $\gamma_{\ell}$ and $\gamma_{\ell+1}$ (Fig. 7). 


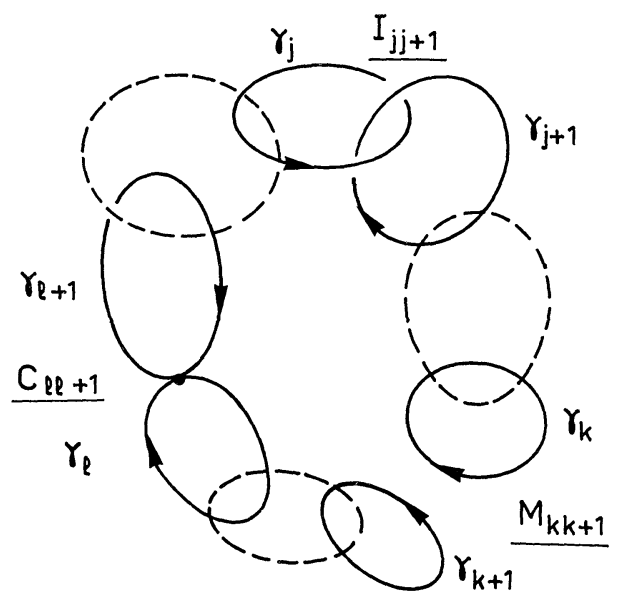

Fig. 7. The different geometrical quantities considered: $I_{j j+1}$ measures the linking number of $\gamma_{j}, \gamma_{j+1}$; $M_{k k+1}$ measures the mutual induction between $\gamma_{k}, \gamma_{k+1} ; C_{\ell \ell+1}$ corresponds to the contact of curves $\gamma_{\ell}, \gamma_{\ell+1}$

The process transforming integral (2.9) into integral (2.28) can be exactly repeated for the present integral (6.8), by using Eq. (6.7). One obtains the analogous formula

$$
\begin{aligned}
& \mathscr{I}\left(\ldots \gamma_{j}, \gamma_{j+1} \ldots \gamma_{k} \sim \gamma_{k+1} \ldots \gamma_{\ell} \cdot \gamma_{\ell+1} \ldots\right) \\
& =\int d^{3} r_{1} \ldots d^{3} r_{n} \delta\left(\mathbf{r}_{1}+\ldots+\mathbf{r}_{n}\right) \\
& \quad \cdot \operatorname{tr}\left[\ldots \Delta_{r_{\ell}} \underset{\ell}{\Gamma}\left(\mathbf{r}_{\ell}\right) \ldots-\underset{\sim}{\Gamma}\left(\mathbf{r}_{k}\right) \ldots \operatorname{curl}_{r_{j} \sim} \Gamma_{j}\left(\mathbf{r}_{j}\right) \ldots\right] .
\end{aligned}
$$

The rules are thus particularly simple: each linking $\gamma_{j}, \gamma_{j+1}$ gives rise to a term curl $\boldsymbol{\Gamma}_{j}$; each mutual induction $\gamma_{k} \sim \gamma_{k+1}$ gives rise to a term $-\boldsymbol{\Gamma}_{k}$; each contact $\gamma_{\ell} \cdot \gamma_{\ell+1}$ gives rise to a term $\Delta \boldsymbol{\Gamma}_{\ell}$. According to definition (2.27) of $\boldsymbol{\Gamma}$, tensor $\Delta \boldsymbol{\Gamma}$ reads for a given curve:

$$
\Delta \underset{\sim}{\boldsymbol{\Gamma}}(\mathbf{r})=\int \frac{d \mathcal{O}}{8 \pi^{2}} \oint \oint d \mathbf{r}_{A} \otimes d \mathbf{r}_{A^{\prime}} \delta\left(\mathbf{r}-\mathbf{r}_{A^{\prime} A}\right)
$$

By using curl curl $=\nabla$ div $-\Delta$ for divergence-free tensor $\Gamma$, we also find for $\Delta \Gamma$ the equivalent expression:

$$
\Delta \underset{\sim}{\boldsymbol{\Gamma}}=-\operatorname{curlcurl} \underset{\sim}{\Gamma}
$$

Our aim is now to establish a factorization theorem for the convolution integral (6.9). We define the Fourier transform $\hat{V}$ of a tensor $V$ by

$$
\hat{\mathbf{V}}(\mathbf{p})=\int d^{3} r e^{i \mathbf{p} \cdot \mathbf{r}} \mathbf{V}(r),
$$

and the convolution integral (6.9) can then be written

$$
\begin{aligned}
& \mathscr{I}\left(\ldots \gamma_{j}, \gamma_{j+1} \ldots \gamma_{k} \sim \gamma_{k+1} \ldots \gamma_{\ell} \cdot \gamma_{\ell+1} \ldots\right) \\
& \quad=\int \frac{d^{3} p}{(2 \pi)^{3}} \operatorname{tr}\left[\ldots \widehat{\sim} \underset{\sim}{\Gamma}(\mathbf{p}) \ldots-\hat{\sim}_{k}(\mathbf{p}) \ldots \widehat{\operatorname{curl}} \prod_{j}(\mathbf{p}) \ldots\right] .
\end{aligned}
$$


The Fourier transforms appearing in (6.13) are related. Indeed we use the expression of the Fourier transform of a curl:

$$
\widehat{\operatorname{curl} \underset{\sim}{\mathbf{V}}}(\mathbf{p})=-i \mathbf{p} \wedge \hat{\mathbf{V}}(\mathbf{p})
$$

and obtain with (6.11):

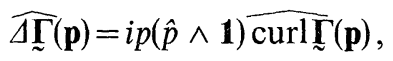

$\hat{\Gamma}$ can be calculated as

$$
\hat{\sim}(\mathbf{p})=-\frac{1}{p^{2}} \widehat{\Delta \Gamma}(\mathbf{p})
$$

Using expression (5.11) of $\widehat{\operatorname{curl} \Gamma}$ in terms of functions $\mathscr{A}$ and $\mathscr{B}$ and applying algebraic rules (5.14), we find

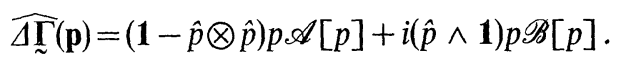

For calculating the trace appearing in the integrand of (6.12), we use isomorphism (5.15). It gives according to (6.15) and (6.16)

$$
\begin{aligned}
\widehat{\operatorname{curl}} \underset{\sim}{\Gamma}(\mathbf{p}) & \rightarrow \varepsilon \mathscr{A}[p]+\mathscr{B}[p] \\
\widehat{\Delta \underset{\sim}{\Gamma}}(\mathbf{p}) & \rightarrow p(\mathscr{A}[p]+\varepsilon \mathscr{B}[p]) \\
-\hat{\Gamma}(\mathbf{p}) & \rightarrow \frac{1}{p}(\mathscr{A}[p]+\varepsilon \mathscr{B}[p]) .
\end{aligned}
$$

We note that, as expected, $\underset{\sim}{\Gamma}(\mathbf{p})$ and $\widehat{\Delta \Gamma(\mathbf{p})}$ have the same parity with respect to $\varepsilon$. It is the opposite of the parity of curl $\Gamma(\mathbf{p})$.

As in Sect. 5, we transform (6.13) by the isomorphism and follow the same procedure as in Eq. (5.17) to (5.22). We obtain in this way the theorem:

Theorem. The integral

$$
\begin{aligned}
& \mathscr{I}\left(\ldots \gamma_{j}, \gamma_{j+1} \ldots \gamma_{k} \sim \gamma_{k+1} \ldots \gamma_{\ell} \cdot \gamma_{\ell+1} \ldots\right) \\
& \quad=\int_{\text {motions }}\left(\ldots I_{j j+1} \ldots M_{k k+1} \ldots C_{\ell \ell+1} \ldots\right)
\end{aligned}
$$

of the cyclic product of linking numbers $I_{j j+1}$ between curves $\gamma_{j}$ and $\gamma_{j+1}$, of mutual inductances $M_{k k+1}$ between curves $\gamma_{k}, \gamma_{k+1}$ and of contacts functions $C_{\ell \ell+1}$ between curves $\gamma_{\ell}, \gamma_{\ell+1}$, over all Euclidean motions (translation and rotations) of the $n$ curves $\gamma_{j}$, is equal to the single integral

$$
\mathscr{I}=\frac{1}{\pi^{2}} \int_{0}^{\infty} d p p^{2} \mathscr{P}_{\text {even }}[p] .
$$

$\mathscr{P}_{\text {even }}[p]$ is the even part of the algebraic product $\mathscr{P}[p]$

$$
\mathscr{P}[p]=\mathscr{P}_{\text {even }}[p]+\varepsilon \mathscr{P}_{\text {odd }}[p],
$$

this product being defined by

$$
\mathscr{P}[p]=\left[\ldots\left(\varepsilon_{\mathscr{A}_{j}}[p]+\mathscr{B}_{j}[p]\right) \ldots \frac{1}{p}\left(\mathscr{A}_{k}[p]+\varepsilon \mathscr{B}_{k}[p]\right) \ldots p\left(\mathscr{A}_{\ell}[p]+\varepsilon \mathscr{B}_{\ell}[p]\right) \ldots\right]
$$


with $\varepsilon^{2}=1$. The product $\mathscr{P}$ is built according to the rules:

Factor $I_{j j+1}$ generates term $\varepsilon_{\mathscr{A}_{j}}[p]+\mathscr{B}_{j}[p]$, where $\mathscr{A}_{j}, \mathscr{B}_{j}$ are characteristic functions of curve $\gamma_{j}$ only.

Factor $M_{k k+1}$ generates term $\frac{1}{p}\left(\mathscr{A}_{k}[p]+\varepsilon \mathscr{B}_{k}[p]\right)$ associated with curve $\gamma_{k}$.

Factor $C_{\ell \ell+1}$ generates factor $p\left(\mathscr{A}_{\ell}[p]+\varepsilon \mathscr{B}_{\ell}[p]\right)$ associated with curve $\gamma_{\ell}^{\prime}$.

With this general result, let us now interpret the odd part $\mathscr{P}_{\text {odd }}[p]=\mathscr{Y}[p]$, obtained together with $\mathscr{P}_{\text {even }}=\mathscr{X}$, when we deal with a complete set of $n$ linkings [see Eq. (5.22b)].

Replacement of a linking $I$ by a mutual inductance $M$ in cyclic product (6.18) changes the parity of product $\mathscr{P}[p](6.18 \mathrm{c})$ and brings in a factor $1 / p$ [rules $(6.18 \mathrm{~d}, \mathrm{e})]$. Likewise, substitution of linking $I$ by contact $C$ changes parity and brings in a factor $p$ [rules $(6.18 \mathrm{~d}, \mathrm{f})]$.

In particular, let us consider the product of $n-1$ linkings and of one mutual inductance. Accordingly:

$$
\begin{aligned}
\mathscr{I}\left(\gamma_{1}, \ldots, \gamma_{n-1}, \gamma_{n} \sim\right) & =\int_{\text {motions }} I_{12} \ldots I_{n-1 n} M_{n 1} \\
& =\frac{1}{\pi^{2}} \int_{0}^{\infty} d p p \mathscr{Y}[p],
\end{aligned}
$$

where $\mathscr{Y}[p]$ is the odd part of $(5.22 \mathrm{~b})$. Likewise, $n-1$ linkings and one contact give:

$$
\begin{aligned}
\mathscr{I}\left(\gamma_{1}, \ldots, \gamma_{n-1}, \gamma_{n} \cdot\right) & =\int_{\text {motions }} I_{12} \ldots I_{n-1 n} C_{n 1} \\
& =\frac{1}{\pi^{2}} \int^{\infty} d p p^{3} \mathscr{Y}[p] .
\end{aligned}
$$

Lastly, let us briefly discuss the convergence of integral (6.18a).

In general, this integral for an arbitrary number of linkings, contacts, and mutual inductances can diverge. Contacts can bring short distance (i.e. $p \rightarrow \infty$ ) divergences. Mutual inductances are long range phenomenas and bring divergences at $p=0$. Compensations between contacts and mutual inductances can occur [cf. rules $(6.18 \mathrm{e}, \mathrm{f})]$, and the convergence must be checked in each particular case, with the help of asymptotic behaviours of $\mathscr{A}, \mathscr{B}$ given in Appendix C. One can check for instance that the integrals $\int C^{2}$ for two curves, $\int C^{3}$ for three curves, both diverge. However, for a larger number $n$ of curves, the integral $\int_{n \geqq 4} C^{n}$ is
convergent

\section{Random Linking, Contact, and Induction of Two Curves}

For two curves, let us consider linking number $I$, mutual inductance $M$, and contact function $C$. We shall give in this section the expression in direct space of the kinematic integrals of products $I M, I C, M C$, and $M^{2}$. These integrals can be 
factorized, like Pohl's kinematic integral of $I^{2}$. New characteristic functions appear.

We could start from the Fourier representation, given by general theorem (6.18) of the last section. For calculations in direct space, it is better to return to the general tensorial expression (6.9). One uses then expressions of tensors $\Gamma, \operatorname{curl} \Gamma$, $\Delta \Gamma$, obtained from results of Sect. 3. We refer to Appendix D for calculations, and give here only the successive generalizations of Pohl's theorem we find.

Theorem. For two curves, all kinematic integrals involving I, M, C can be factorized as follows

$$
\int M I=2 \pi \int_{0}^{\infty} d r\left[\mathscr{A}_{1}^{+}(r) \mathscr{B}_{2}(r)+\mathscr{A}_{2}^{+}(r) \mathscr{B}_{1}(r)\right],
$$

where the characteristic function $\mathscr{A}^{+}$is defined by

$$
\begin{aligned}
\mathscr{A}^{+}(r) & =r \int_{r}^{\infty} d r^{\prime} \frac{1}{r^{\prime}} \mathscr{A}\left(r^{\prime}\right), \\
\int C I & =2 \pi \int_{0}^{\infty} \frac{d r}{r}\left\{\frac{d}{d r}\left[r \mathscr{A}_{1}(r)\right] \mathscr{B}_{2}(r)+\frac{d}{d r}\left[r \mathscr{A}_{2}(r)\right] \mathscr{B}_{1}(r)\right\}, \\
\int C M & \equiv \int I^{2}=2 \pi \int_{0}^{\infty} d r\left[\mathscr{A}_{1}(r) \mathscr{A}_{2}(r)+\mathscr{B}_{1}(r) \mathscr{B}_{2}(r)\right] .
\end{aligned}
$$

On the other hand:

$$
\int C^{2} \text { diverges. }
$$

Finally

$$
\int M^{2}=2 \pi \int_{0}^{\infty} d r\left[\mathscr{A}_{1}^{+}(r) \mathscr{A}_{2}^{+}(r)+\mathscr{B}_{1}^{+}(r) \mathscr{B}_{2}^{+}(r)\right]
$$

where the function $\mathscr{B}^{+}$is the particular primitive of $\mathscr{B}$ :

$$
\mathscr{B}^{+}(r)=\int_{r}^{\infty} d r^{\prime} \mathscr{B}\left(r^{\prime}\right)
$$

Identity (7.4) can be checked immediately with the help of rules of Theorem (6.18) in Fourier space.

This completes the set of theorems generalizing Pohl's formula.

\section{Conclusion}

In this article, we have introduced a compact tensorial formalism. It enabled us to describe random linking of closed rigid curves and their transformation properties under translation and rotations of these curves. We showed that each curve can be characterized by a single mathematical object, its tensor curl $\Gamma$. The geometrical expression of this tensor was obtained by direct application of Gauss' theorem of potential theory. This elucidated the special form of the result. For two curves, 
Pohl's formula, extended by des Cloizeaux and Ball, was thus directly demonstrated.

For $n$ curves, arranged in a ring, the cyclic product of their linking numbers $I$ is the natural generalization of the squared quantity $I^{2}$ for two curves. We calculated the kinematic integral of this linking product over the group of motions of these $n$ curves. We obtained its factorization, in Fourier space, in terms of characteristic functions. This factorization exists in commutative algebra of two elements $1, \varepsilon$ with $\varepsilon^{2}=1$. The characteristic functions are coefficients of $1, \varepsilon$.

A second natural generalization was found. We showed that the preceding algebra enables us to calculate a whole family of integrals over the group of motions of rigid curves. For that purpose we defined the contacts and mutual inductances of curves, which are intimately related to Gauss linking numbers.

A general factorization theorem in terms of characteristic functions was given. It allows us to calculate the kinematic integral of any cyclic product, the factors of which are linking numbers, contact functions or mutual inductances.

These new mathematical results belong to topology and, in a stronger way, to potential theory. By their relation to topology they could be useful for a theory of polymer rings, yet to come. They could also be useful mathematical theorems for electromagnetism.

\section{Appendix A}

Proof of Lemma (3.3). We shall prove that any tensor $\Gamma(\mathbf{r})$, depending on only one vector $\mathbf{r}$, and having a vanishing divergence, necessarily reads

$$
\Gamma(\mathbf{r})=\operatorname{curl} \operatorname{curl} \varphi(r) \mathbf{1}+\operatorname{curl} \psi(r) \mathbf{1},
$$

$\varphi$ and $\psi$ are functions of $r=|\mathbf{r}|$ and $\mathbf{1}$ is the unity matrix. Curl acts on left indices.

We first note that a tensor $\underset{\sim}{\Gamma}$, function of a single vector $\mathbf{r}$, must be of the general form

$$
\Gamma(\mathbf{r})=\mathbf{r} \otimes \mathbf{r} f(r)+\mathbf{1} g(r)+(\mathbf{r} \wedge \mathbf{1}) h(r),
$$

where $f, g, h$ are arbitrary functions of modulus $r$. It is easy to check the equivalent general form:

$$
\underset{\sim}{\Gamma}(\mathbf{r})=\nabla \otimes \nabla \varphi_{1}(r)+\mathbf{1} \Delta \varphi_{2}(r)+\nabla \psi(r) \wedge \mathbf{1} .
$$

$\varphi_{1}$ and $\varphi_{2}$ are related to $f$ and $g$ by second order differential equations. $\psi$ is related to $h$ by $\frac{1}{r} \frac{d}{d r} \psi=h$. We remark that

$$
\nabla \psi(r) \wedge \mathbf{1}=\operatorname{curl}(\psi(r) \mathbf{1}) .
$$

Then the left divergence of the tensor (A.3) is the vector

$$
\begin{aligned}
\operatorname{div} \underset{\sim}{\Gamma}(\mathbf{r}) & =\nabla^{2}\left(\nabla \varphi_{1}(r)\right)+\nabla \cdot \mathbf{1} \Delta \varphi_{2}(r) \\
& =\nabla\left[\Delta \varphi_{1}(r)+\Delta \varphi_{2}(r)\right]=\mathbf{0},
\end{aligned}
$$


which must vanish, according to our assumptions. Thus, we have $\Delta\left(\varphi_{1}+\varphi_{2}\right)=C$, where $C$ is a constant. Eliminating $\varphi_{2}$ in (A.3), we get for $\Gamma$ :

$$
\underset{\sim}{\Gamma}=(\nabla \otimes \nabla-1 \Delta) \varphi_{1}+C \mathbf{1}+\operatorname{curl} \psi \mathbf{1} \text {. }
$$

$C 1$ can be written $C 1=(\nabla \otimes \nabla-1 \Delta)\left(-C / 4 r^{2}\right)$. Thus, defining $\varphi=\varphi_{1}-C / 4 r^{2}$, we get:

$$
\underset{\sim}{\Gamma}=(\nabla \otimes \nabla-1 \Delta) \varphi+\operatorname{curl} \psi \mathbf{1}
$$

Using

$$
(\boldsymbol{\nabla} \otimes \nabla-\mathbf{1} \Delta) \varphi=(\boldsymbol{\nabla d i v}-\Delta)(\varphi \mathbf{1})=\operatorname{curl} \operatorname{curl}(\varphi \mathbf{1}),
$$

we obtain (A.7) in form (A.1). Q.E.D.

\section{Appendix B}

An Equivalent $\mathscr{B}$ Function for two Curves. We consider for two curves the contribution of $\mathscr{B}$ functions to the kinematic integral:

$$
\mathscr{I}_{(B)}\left(\gamma_{1}, \gamma_{2}\right)=2 \pi \int_{0}^{\infty} d r \mathscr{B}_{1}(r) \mathscr{B}_{2}(r)
$$

where $\mathscr{B}$ reads :

$$
\mathscr{B}(r)=\frac{r}{4 \pi} \oint \oint\left(\frac{\mathbf{r}_{A A^{\prime}}}{r_{A A^{\prime}}^{3}}, d \mathbf{r}_{A}, d \mathbf{r}_{A^{\prime}}\right) \theta\left(r_{A A^{\prime}}-r\right) .
$$

For computing integral $\mathscr{I}_{(B)}$ for two curves, another possible function $\mathscr{B}$ is [4]:

$$
\mathscr{B}(r)=\frac{1}{4 \pi r^{2}} \oint \oint\left(\mathbf{r}_{A A^{\prime}}, d \mathbf{r}_{A}, d \mathbf{r}_{A^{\prime}}\right) \theta\left(r-r_{A A^{\prime}}\right),
$$

such that

$$
\mathscr{I}_{(B)}\left(\gamma_{1}, \gamma_{2}\right)=2 \pi \int_{0}^{\infty} d r^{\prime} \mathscr{B}_{1}\left(r^{\prime}\right) \mathscr{B}_{2}\left(r^{\prime}\right)
$$

This can be checked by performing, inside integral (B.1), the local change of variable $r \rightarrow r^{\prime}$ such that

$$
r r^{\prime}=r_{A_{1} A_{1}^{\prime}} r_{A_{2} A_{2}^{\prime}} \text {. }
$$

It corresponds to an inversion with respect to the sphere of radius $\left(r_{A_{1} A_{1}^{\prime}} r_{A_{2} A_{2}^{\prime}}\right)^{1 / 2}$.

\section{Appendix C}

Characteristic Functions $\mathscr{A}[p], \mathscr{B}[p]$. These functions are defined by

$$
\begin{aligned}
& \mathscr{A}[p]=-2 \pi \frac{d}{d p} \int_{0}^{\infty} d r \frac{\sin p r}{p r} \mathscr{A}(r), \\
& \mathscr{B}[p]=\frac{2 \pi}{p} \int_{0}^{\infty} d r \sin p r \mathscr{B}(r) .
\end{aligned}
$$


$\mathscr{A}(r), \mathscr{B}(r)$ are given by:

$$
\begin{aligned}
& \mathscr{A}(r)=\frac{1}{4 \pi r} \oint \oint d \mathbf{r}_{A} \cdot d \mathbf{r}_{A^{\prime}} \theta\left(r-r_{A A^{\prime}}\right), \\
& \mathscr{B}(r)=-\frac{r}{4 \pi} \oint \oint\left(\nabla_{A} \frac{1}{r_{A A^{\prime}}}, d \mathbf{r}_{A}, d \mathbf{r}_{A^{\prime}}\right) \theta\left(r_{A A^{\prime}}-r\right) .
\end{aligned}
$$

Define

$$
a[p]=\frac{d}{d p} \int_{0}^{\infty} \frac{d r}{r} \frac{\sin p r}{p r} \theta\left(r-r_{A A^{\prime}}\right) .
$$

Differentiating inside the integral:

$$
a[p]=\int_{0}^{\infty} d r \frac{1}{p} \frac{d}{d r}\left(\frac{\sin p r}{p r}\right) \theta\left(r-r_{A A^{\prime}}\right) .
$$

Integrating by parts :

$$
a[p]=\frac{1}{p}\left[\frac{\sin p r}{p r} \theta\left(r-r_{A A^{\prime}}\right)\right]_{0}^{\infty}-\frac{1}{p} \frac{\sin p r_{A A^{\prime}}}{p r_{A A^{\prime}}} .
$$

The first term vanishes. Thus, one gets

$$
\begin{aligned}
\mathscr{A}[p] & =-\frac{1}{2} \oint \oint d \mathbf{r}_{A} \cdot d \mathbf{r}_{A^{\prime}} a[p] \\
& =\frac{1}{2 p} \oint \oint d \mathbf{r}_{A} \cdot d \mathbf{r}_{A^{\prime}} \frac{\sin p r_{A A^{\prime}}}{p r_{A A^{\prime}}},
\end{aligned}
$$

which is similar to another function $\mathscr{A}[p]$ used in [4].

Define, for calculating $\mathscr{B}[p]$ :

$$
b[p]=\int_{0}^{\infty} d r \sin (p r) r \theta\left(r_{A A^{\prime}}-r\right) .
$$

It reads

$$
b[p]=-\frac{d}{d p} \int_{0}^{\infty} d r \cos p r \theta\left(r_{A A^{\prime}}-r\right) .
$$

Integrating by parts

$$
\begin{aligned}
b[p] & =-\frac{d}{d p}\left\{\left[\frac{\sin p r}{p} \theta\left(r_{A},-r\right)\right]_{0}^{\infty}-\frac{\sin p r_{A A^{\prime}}}{p}\right\} \\
& =\frac{d}{d p}\left(\frac{1}{p} \sin p r_{A A^{\prime}}\right) .
\end{aligned}
$$

Thus

$$
\mathscr{B}[p]=-\frac{1}{2 p} \oint \oint\left(\nabla_{A} \frac{1}{r_{A A^{\prime}}}, d \mathbf{r}_{A}, d \mathbf{r}_{A^{\prime}}\right) \frac{d}{d p}\left[\frac{1}{p}\left(\sin p r_{A A^{\prime}}\right)\right],
$$

which is similar to another function $\mathscr{B}$ used in [4]. 


\section{Asymptotic Limits}

For $p \rightarrow 0$. We use definitions (C.1) and (C.2). Expanding the sin function, one gets

$$
\begin{aligned}
& \mathscr{A}[p]=\mathscr{A}_{0} p \quad(p \rightarrow 0) \quad \text { with } \quad \mathscr{A}_{0}=\frac{2 \pi}{3} \int_{0}^{\infty} d r r^{2} \mathscr{A}(r) \\
& \mathscr{B}[0]=2 \pi \int_{0}^{\infty} d r \mathscr{B}(r) \equiv \mathscr{B}_{0}<\infty .
\end{aligned}
$$

$\mathscr{A}_{0}$ and $\mathscr{B}_{0}$ are finite constants.

For $p \rightarrow \infty$. By a change of variable:

$$
\mathscr{A}[p]=-2 \pi \frac{d}{d p} \int_{0}^{\infty} d x \frac{1}{p} \frac{\sin x}{x} \mathscr{A}(x / p),
$$

and therefore, for $p \rightarrow \infty$, using $\int_{0}^{\infty} d x \frac{\sin x}{x}=\frac{\pi}{2}$ :

$$
\mathscr{A}[p]=\pi^{2} \mathscr{A}(0) \frac{1}{p^{2}} \quad(p \rightarrow \infty) .
$$

For function $\mathscr{B}$, one uses the explicit expression (C.6). There clearly exists a majorant $\mathscr{B}_{\infty}$ such that

$$
|\mathscr{B}[p]|<\frac{\mathscr{B}_{\infty}}{p^{2}} \quad(p \rightarrow \infty) .
$$

One can then verify the convergence of integral (5.22a). The integrand $p^{2} \mathscr{X}[p]$, defined by (5.22b) is regular for $p=0$, because of (C.7). For $p \rightarrow \infty,($ C.8) and (C.9) give $p^{2} p^{-2 n}$ as a majorant of $\left|p^{2} \mathscr{X}[p]\right|$. Thus integral $\mathscr{I}$ of $n$ linkings is absolutely convergent.

\section{Appendix D}

Linking, Contact, and Inductance of two Curves. We shall calculate for two curves the kinematic integrals of products $M I, C I$, and $M^{2}$. The integral of $C^{2}$ is not defined. According to (6.9), these integrals read:

$$
\begin{aligned}
\int M I & =-\int d^{3} r \operatorname{tr}\left[\operatorname{curl} \sim_{\sim}(-\mathbf{r}) \Gamma_{1}(\mathbf{r})\right], \\
\int C I & =\int d^{3} r \operatorname{tr}\left[\operatorname{curl} \Gamma_{\sim}(-\mathbf{r}) \Delta \underset{\sim}{\Gamma_{1}}(\mathbf{r})\right], \\
\int M^{2} & =\int d^{3} r \operatorname{tr}\left[\underset{\sim}{\Gamma_{2}}(-\mathbf{r}) \Gamma_{1}(\mathbf{r})\right] .
\end{aligned}
$$

Section 3 gives the successive tensors

$$
\begin{aligned}
\underset{\Gamma}{\Gamma} & =(\nabla \otimes \nabla-\mathbf{1} \Delta) \varphi+\nabla \psi \wedge \mathbf{1}, \\
\operatorname{curl} \underset{\sim}{\Gamma} & =-\nabla(\Delta \varphi) \wedge \mathbf{1}+(\nabla \otimes \nabla-\mathbf{1} \Delta) \psi, \\
\Delta \underset{\sim}{\Gamma} & =(\nabla \otimes \nabla-\mathbf{1} \Delta) \Delta \varphi+\nabla(\Delta \psi) \wedge \mathbf{1} .
\end{aligned}
$$


Let us recall the relations between auxiliary functions $\varphi(r), \psi(r)$ and functions $\mathscr{A}(r), \mathscr{B}(r) . \varphi$ and $\mathscr{A}$ are related by Eqs. (3.12) and (3.24):

$$
\frac{d}{d r}(\Delta \varphi(r))=-\frac{1}{2 r} \mathscr{A}(r) \text {. }
$$

The solution, which must vanish at infinity, is then given by:

$$
\Delta \varphi(r)=\frac{1}{2 r} \mathscr{A}^{+}(r)
$$

where we set by definition:

$$
\mathscr{A}^{+}(r)=r \int_{r}^{\infty} d r^{\prime} \frac{1}{r^{\prime}} \mathscr{A}\left(r^{\prime}\right)
$$

on the other hand, $\psi$ and $\mathscr{B}$ are related by Eq. (3.28)

$$
\Delta \psi(r)=-\frac{1}{2 r} \mathscr{B}(r) .
$$

$\psi$ appears in tensor $\underset{\sim}{\Gamma}$ (D.4), through gradient term $\nabla \psi$ only.

$\psi$ is thus defined up to a constant. Searching for a solution vanishing at infinity, as $\mathscr{B}$ does, we solve (D.10) by using the Laplacian for spherical coordinates $\Delta=\frac{1}{r} \frac{d^{2}}{d r^{2}} r$, valid everywhere, except at the origin. One finds easily

$$
\psi(r)=-\frac{1}{2 r}\left[\mathscr{B}^{++}(r)-\mathscr{B}^{++}(0)\right]
$$

where $\mathscr{B}^{++}$is the second primitive:

$$
\mathscr{B}^{++}(r)=\int_{r}^{\infty} d r^{\prime} \mathscr{B}^{+}\left(r^{\prime}\right), \quad \mathscr{B}^{+}(r)=\int_{r}^{\infty} d r^{\prime} \mathscr{B}\left(r^{\prime}\right) .
$$

The subtraction of the principal part at $r=0$ in (D.11) insures that $\Delta \psi$ does not contain any distribution at $r=0$. We shall need two last auxiliary formulae, already used in the text:

$$
\int d^{3} r \operatorname{tr}[(\boldsymbol{\nabla} \otimes \nabla-\mathbf{1} \Delta) f(\boldsymbol{\nabla} \otimes \nabla-\mathbf{1} \Delta) g]=2 \int d^{3} r \Delta f \Delta g,
$$

and

$$
\int d^{3} r \operatorname{tr}[(\nabla f \wedge \mathbf{1})(\nabla g \wedge \mathbf{1})]=-2 \int d^{3} r \nabla f \cdot \nabla g
$$

Equation (D.1) can then be rewritten, with the help of (D.4) and (D.5)

$$
\int M I=-2 \int d^{3} r\left[\Delta \psi_{2} \Delta \varphi_{1}-\nabla\left(\Delta \varphi_{2}\right) \cdot \nabla \psi_{1}\right],
$$

where one must take $(\nabla f)(-\mathbf{r})=-\nabla f(r)$. Then, by integrating by parts, one gets

$$
\int M I=-2 \int d^{3} r\left(\Delta \varphi_{1} \Delta \psi_{2}+\Delta \varphi_{2} \Delta \psi_{1}\right),
$$


which gives [see (D.8) and D.10)] the announced result:

$$
\int M I=2 \pi \int d r\left[\mathscr{A}_{1}^{+}(r) \mathscr{B}_{2}(r)+\mathscr{A}_{2}^{+}(r) \mathscr{B}_{1}(r)\right] .
$$

In the same way, one obtains

$$
\begin{aligned}
\int C I & =2 \int d^{3} r\left[\Delta \psi_{2} \Delta^{2} \varphi_{1}-\nabla\left(\Delta \varphi_{2}\right) \cdot \nabla\left(\Delta \psi_{1}\right)\right] \\
& =-2 \int d^{3} r\left[\nabla\left(\Delta \psi_{2}\right) \cdot \nabla\left(\Delta \varphi_{1}\right)+\nabla\left(\Delta \varphi_{2}\right) \cdot \nabla\left(\Delta \psi_{1}\right)\right] .
\end{aligned}
$$

Using Eqs. (D.7) and (D.10) :

$$
\int C I=-2 \pi \int_{0}^{\infty} d r\left\{r \mathscr{A}_{1}(r) \frac{d}{d r}\left[\frac{\mathscr{B}_{2}}{r}(r)\right]+r \mathscr{A}_{2}(r) \frac{d}{d r}\left[\frac{\mathscr{B}_{1}}{r}(r)\right]\right\} .
$$

Integrating by parts gives (7.3). We finally calculate (D.3):

$$
\int M^{2}=2 \int d^{3} r\left(\Delta \varphi_{1} \Delta \varphi_{2}+\nabla \psi_{1} \cdot \nabla \psi_{2}\right) .
$$

The second part of this integral can be calculated by using (D.10) and (D.11):

$$
-2 \int d^{3} r\left(\Delta \psi_{1}\right) \psi_{2}=2 \pi \int_{0}^{\infty} d r\left[\mathscr{B}_{1}^{++}(r)-\mathscr{B}_{1}^{++}(0)\right] \mathscr{B}_{2}(r)
$$

and, by integrating by parts [use (D.12)]:

$$
=2 \pi\left[\mathscr{B}_{2}^{+}(r)\left[\mathscr{B}_{1}^{++}(r)-\mathscr{B}_{1}^{++}(0)\right]\right]_{0}^{\infty}+2 \pi \int_{0}^{\infty} d r \mathscr{B}_{2}^{+}(r) \mathscr{B}_{1}^{+}(r) .
$$

The first term vanishes, and one gets for (D.16) and (D.8), the announced result:

$$
\int M^{2}=2 \pi \int_{0}^{\infty} d r\left[\mathscr{A}_{1}^{+}(r) \mathscr{A}_{2}^{+}(r)+\mathscr{B}_{1}^{+}(r) \mathscr{B}_{2}^{+}(r)\right] .
$$

Acknowledgements. I thank J. des Cloizeaux and R. Balian for interesting discussions.

\section{References}

1. Gauss, C.F.: Complete Works, fifth band, Königliche Gesellschaft der Wissenschaften zu Göttingen, 1867, p. 605

Alexandroff, P., Hopf, H.: Topologie I. Berlin: Springer 1935

Rolfsen, D. : Knots and Links. In: Mathematics Lecture, Ser. 7. Berkeley, CA : Publish or Perish Inc. 1976 , p. 132

2. Kellogg, O.D. : Foundations of Potential Theory. Berlin, Heidelberg, New York : Springer 1967

3. Pohl, W.F.: International Symposium in honour of N. H. Kuiper, Utrecht 1980. In: Lecture Notes in Mathematics, Berlin, Heidelberg, New York: Springer 1981

4. Des Cloizeaux, J., Ball, R.: Commun. Math. Phys. 80, 543-553 (1981)

5. Neumann, F.: Abhandl. Preuss. Akad., 1845. Reprinted in Ostwald's Klassiker No. 10; See also Sommerfeld, A.: Electrodynamics, p. 105. New York: Academic Press 1952

Communicated by J. L. Lebowitz

Received March 2, 1981

Note added in proof. The generalization of the results to the linking numbers of manifolds in $\mathbb{E}^{n}$ (B. Duplantier, Saclay preprint DPh-T/81-61) will be given in a forthcoming paper. 